\title{
BOUND ON THE MAXIMAL FUNCTION ASSOCIATED TO THE LAW OF THE ITERATED LOGARITHMS FOR BERNOULLI RANDOM FIELDS
}

\author{
DAVIDE GIRAUDO
}

\begin{abstract}
We provide a sufficient condition for the bounded law of the iterated logarithms for strictly stationary random fields expressable as a functional of i.i.d. random fields when the summation is done on rectangles. The study is done via the control of the moments of an appropriated maximal function. Applications to functionals of linear random fields, functions of a Gaussian linear random field and Volterra process are given.
\end{abstract}

\section{Goal of the Paper And main Results}

1.1. Bounded law of the iterated logarithms for random fields. Let $d \geqslant 1$ be an integer and let $\left(X_{i}\right)_{i \in \mathbb{Z}^{d}}$ be a random field and denote the partial sums

$$
S_{n}:=\sum_{1 \preccurlyeq i \preccurlyeq n} X_{i}, \quad n \succcurlyeq 1,
$$

where $\preccurlyeq$ denotes the coordinatewise order on the elements of $\mathbb{Z}^{d}$, that is, for $\boldsymbol{i}=\left(i_{q}\right)_{q=1}^{d}$ and $\boldsymbol{j}=$ $\left(j_{q}\right)_{q=1}^{d}, \boldsymbol{i} \preccurlyeq \boldsymbol{j}$ if $i_{q} \leqslant j_{q}$ for all $q \in[d]:=\{1, \ldots, d\}$ (and similarly, we write $\boldsymbol{i} \succcurlyeq \boldsymbol{j}$ if $i_{q} \geqslant j_{q}$ for all $q \in[d])$ and $\mathbf{1}=(1, \ldots, 1)$. The understanding of the behavior of such partial sums has received attention in the past years. When the partial sums (1.1.1) are normalized by $|\boldsymbol{n}|=\prod_{i=1}^{d} n_{i}$ and the random field $\left(X_{i}\right)_{i \in \mathbb{Z}^{d}}$ is strictly stationary, functional central limit theorems have been established under various dependence structures: martingale differences with respect to the lexicographic order [Ded01, Coh16], martingale differences [BD79, PR198], orthomartingale differences and via orthomartingale approximation (see [VW14, CDV15, Vol15, PZ18b, PZ18a]).

Concerning the law of the iterated logarithms, it has been shown in [Wic73] that for an i.i.d. collection of centered random variables $\left(X_{\boldsymbol{i}}\right)_{\boldsymbol{i} \in \mathbb{Z}^{d}}$, (with $d>1$ ) the following equivalence holds:

$$
\begin{aligned}
\mathbb{E}\left[X_{\mathbf{0}}^{2}\left(L\left(\left|X_{\mathbf{0}}\right|\right)\right)^{d-1} / L L\left(\left|X_{\mathbf{0}}\right|\right)\right]<+\infty & \\
& \Leftrightarrow \limsup _{\boldsymbol{n} \rightarrow+\infty} \frac{1}{\sqrt{|\boldsymbol{n}| L L(|\boldsymbol{n}|)}} S_{\boldsymbol{n}}=\left\|X_{\mathbf{0}}\right\|_{2} \sqrt{d}=-\liminf _{\boldsymbol{n} \rightarrow+\infty} \frac{1}{\sqrt{|\boldsymbol{n}| L L(|\boldsymbol{n}|)}} S_{\boldsymbol{n}},
\end{aligned}
$$

where $L:(0,+\infty) \rightarrow \mathbb{R}$ is defined by $L(x)=\max \{\ln x, 1\}$ and $L L:(0,+\infty) \rightarrow \mathbb{R}$ by $L L(x)=$ $L \circ L(x)$, and for a family of numbers $\left(a_{\boldsymbol{n}}\right)_{\boldsymbol{n} \succcurlyeq \mathbf{1}}, \lim \sup _{\boldsymbol{n} \rightarrow+\infty} a_{\boldsymbol{n}}:=\lim _{m \rightarrow+\infty} \sup _{\boldsymbol{n} \succcurlyeq m \mathbf{1}} a_{\boldsymbol{n}}$ and similarly for lim inf.

In particular, the moment condition as well as the limsup/lim inf depend on the dimension $d$ and the normalization by $\sqrt{|\boldsymbol{n}| L L(|\boldsymbol{n}|)}$ is the best possible among those guaranting the

Date: May 17, 2021.

2010 Mathematics Subject Classification. 60G60; 60G10.

Key words and phrases. random fields, bounded law of the iterated logarithms. 
finiteness of the limsup/liminf in (1.1.2). Some related results have been obtained under other dependence structures; see [Sha15, Sha06, Bu195, Jia99] for instance.

In general, finding $\limsup _{\boldsymbol{n} \rightarrow+\infty} \frac{1}{\sqrt{|\boldsymbol{n}| L L(|\boldsymbol{n}|)}} S_{\boldsymbol{n}}$ is a hard task. Another one consists in establishing finiteness of $\sup _{\boldsymbol{n} \in \mathbb{N}^{d}} \frac{1}{\sqrt{|\boldsymbol{n}| L L(|\boldsymbol{n}|)}}\left|S_{\boldsymbol{n}}\right|$ (where $\mathbb{N}$ denotes the set of positive integers) or integrability of this random variable. More precisely, we would like to find a sufficient condition on the moments and the dependence of a stationary random field such that the quantity

$$
\left\|\sup _{\boldsymbol{n} \in \mathbb{N}^{d}} \frac{1}{\sqrt{|\boldsymbol{n}| L L(|\boldsymbol{n}|)}}\left|S_{\boldsymbol{n}}\right|\right\|_{p}<+\infty, 1<p<2,
$$

is finite. When $d=1$ and $\left(X_{i}\right)_{i \geqslant 1}$ is i.i.d. centered, it has been shown in [Pis76] that for $1<p<2$,

$$
\left\|\sup _{n \geqslant 1} \frac{1}{\sqrt{n L L(n)}}\left|\sum_{i=1}^{n} X_{i}\right|\right\|_{p} \leqslant c_{p}\left\|X_{1}\right\|_{2} .
$$

This has been extended to martingales in [Cun15], and to higher moments in [MP06].

In this paper, we will be concentrated in the following questions. First, we would like to give bound on the quantity involved in (1.1.3) in the i.i.d. case. Results in the one dimensional case are known, but to the best of our knowledge, it seems that no results are available in dimension greater than one. Once this is done for i.i.d. random fields, a similar question can be treated for a strictly stationary random field which can be expressed as a functional of finitely many i.i.d. random variables, and then extend this to more general random fields, which are functionals of an i.i.d. collection of random variable indexed by $\mathbb{Z}^{d}$.

1.2. Main results. We assume that $X_{\boldsymbol{i}}$ has the form $f\left(\left(\varepsilon_{\boldsymbol{i}-\boldsymbol{j}}\right)_{\boldsymbol{j} \in \mathbb{Z}^{d}}\right)$ where $f: \mathbb{R}^{\mathbb{Z}^{d}} \rightarrow \mathbb{R}$ is measurable (with $\mathbb{R}^{\mathbb{Z}^{d}}$ endowed with the product topology) and $\left(\varepsilon_{\boldsymbol{u}}\right)_{\boldsymbol{u} \in \mathbb{Z}^{d}}$ is an independent identically distributed random field. This class of random fields has been studied during the previous years, including a lot of work in the area of functional central limit theorems [EVW13,BD14,KVW16]. When the summation on rectangles is considered, moments of order two are sufficient and moment of order $p>2$ can be required for other types of partial sum process.

The condition for the control of the maximal function associated to the law of the iterated logarithms involved in (1.1.3) will require slightly more than finite moments of order 2 . In order to state it, we define for $p>1$ and $r \geqslant 0$, the function $\varphi_{p, r}:[0,+\infty)$ by $\varphi_{p, r}(x):=$ $x^{p}(1+\log (1+x))^{r}$ and denote by $\mathbb{L}_{p, r}$ the Orlicz space associated to this function. We define the norm $\|\cdot\|_{p, r}$ of an element $X$ of $\mathbb{L}_{p, r}$ by

$$
\|X\|_{p, r}:=\inf \left\{\lambda>0 \mid \mathbb{E}\left[\varphi_{p, r}\left(\frac{X}{\lambda}\right)\right] \leqslant 1\right\} .
$$

Denote also for $\boldsymbol{i}=\left(i_{q}\right)_{q=1}^{d}$ the quantity $\|\boldsymbol{i}\|_{\infty}:=\max _{1 \leqslant q \leqslant d}\left|i_{q}\right|$ and $\mathbf{0}:=(0, \ldots, 0)$.

Theorem 1.1. Let $\left(X_{i}\right)_{i \in \mathbb{Z}^{d}}$ be a centered random field such that there exist an i.i.d. collection of random variables $\left\{\varepsilon_{\boldsymbol{u}}, \boldsymbol{u} \in \mathbb{Z}^{d}\right\}$ and a measurable function $f: \mathbb{R}^{\mathbb{Z}^{d}} \rightarrow \mathbb{R}$ such that $X_{\boldsymbol{i}}=$ 
$f\left(\left(\varepsilon_{\boldsymbol{i}-\boldsymbol{j}}\right)_{\boldsymbol{j} \in \mathbb{Z}^{d}}\right)$. For all $1<p<2$, the following inequality holds:

$$
\left\|\sup _{\boldsymbol{n} \in \mathbb{N}^{d}} \frac{1}{\sqrt{|\boldsymbol{n}| L L(|\boldsymbol{n}|)}} \mid \sum_{\mathbf{1} \preccurlyeq \boldsymbol{i} \preccurlyeq \boldsymbol{n}} X_{\boldsymbol{i}}\right\|\left\|_{p} \leqslant c_{p, d} \sum_{j \geqslant 0}(j+1)^{d / 2}\right\| X_{\mathbf{0}, j} \|_{2, d-1},
$$

where $c_{p, d}$ depends only on $p$ and $d$ and

$$
\begin{gathered}
X_{\mathbf{0}, j}=\mathbb{E}\left[X_{\mathbf{0}} \mid \sigma\left\{\varepsilon_{\boldsymbol{u}},\|\boldsymbol{u}\|_{\infty} \leqslant j\right\}\right]-\mathbb{E}\left[X_{\mathbf{0}} \mid \sigma\left\{\varepsilon_{\boldsymbol{u}},\|\boldsymbol{u}\|_{\infty} \leqslant j-1\right\}\right], \quad j \geqslant 1 ; \\
X_{\mathbf{0}, 0}:=\mathbb{E}\left[X_{\mathbf{0}} \mid \sigma\left\{\varepsilon_{\mathbf{0}}\right\}\right] .
\end{gathered}
$$

Observe that by the martingale convergence theorem, the sequence $\left(\left\|X_{\mathbf{0}, j}\right\|_{2, d-1}\right)_{j \geqslant 1}$ converges to 0 provided that $X_{\mathbf{0}} \in \mathbb{L}_{2, d-1}$.

We will now formulate a result in the spirit of Theorem 1.1 for subsets which can be expressed as a finite union of disjoint rectangles. We first present a result for i.i.d. random fields where the summation is done on subset of $\mathbb{Z}^{d}$ under an assumption on the cardinal. A central limit theorem for linear random fields has been established in [MW11] and a local central limit theorem in [FPS20]. The case of short and long range dependent random field were adressed. In the short range dependent case, that is, $\sum_{\boldsymbol{i} \in \mathbb{Z}^{d}}\left|a_{\boldsymbol{i}}\right|<\infty$, no condition on the size of the summation subset was made. However, one needs a bound on the size of the sets for the control of the maximal function, even in the case $d=1$. Indeed, taking $\Lambda_{n}$ pairwise disjoint and and i.i.d. $\left(X_{\boldsymbol{i}}\right)_{\boldsymbol{i} \in \mathbb{Z}^{d}}$ where $X_{\boldsymbol{i}}$ is standard normal, the random variable $\sup _{n \geqslant 1} \frac{1}{\sqrt{\ell_{n} L L\left(\ell_{n}\right)}}\left|\sum_{i \in \Lambda_{n}} X_{i}\right|$ has the same distribution as $\sup _{n \geqslant 1} \frac{1}{\sqrt{L L\left(\ell_{n}\right)}}\left|N_{n}\right|$, where $\left(N_{n}\right)_{n \geqslant 1}$ is an i.i.d. sequence of standard normal random variables. Therefore, by the second Borel-Cantelli lemma, the random variable $\sup _{n \geqslant 1} \frac{1}{\sqrt{\ell_{n} L L\left(\ell_{n}\right)}}\left|\sum_{i \in \Lambda_{n}} X_{i}\right|$ is almost surely finite if and only if for some $M, \sum_{n \geqslant 1} \mathbb{P}\left\{\left|N_{n}\right|>M \sqrt{L L\left(\ell_{n}\right)}\right\}$ is finite. Using a lower tail inequality for the distribution function of a normal random variable, this imposes the convergence of the series $\sum_{n \geqslant 1} \exp \left(-M^{2} L\left(\ell_{n}\right) / 2\right) / \sqrt{L L\left(\ell_{n}\right)}$.

Theorem 1.2. Let $\left(\varepsilon_{\boldsymbol{u}}\right)_{\boldsymbol{u} \in \mathbb{Z}^{d}}$ be an i.i.d. centered random field having a finite variance, let $\left(a_{\boldsymbol{i}}\right)_{\boldsymbol{i} \in \mathbb{Z}^{d}}$ be an absolutely summable sequence of integers and denote by $\left(X_{\boldsymbol{i}}\right)_{\boldsymbol{i} \in \mathbb{Z}^{d}}$ the linear process given by $X_{\boldsymbol{i}}:=\sum_{\boldsymbol{j} \in \mathbb{Z}^{d}} a_{\boldsymbol{j}} \varepsilon_{\boldsymbol{i}-\boldsymbol{j}}$. Let $\left(\Lambda_{n}\right)_{n \geqslant 1}$ be a sequence of finite subsets of $\mathbb{Z}^{d}$ and denote by $\ell_{n}$ the cardinal of $\Lambda_{n}$. Suppose that there exists $\delta>0$ such that $\ell_{n+1} \geqslant \ell_{n} \geqslant \exp \left(n^{\delta}\right)$ and $C>0$ such that $\sum_{k=1}^{n} \sqrt{\ell_{k} / L L\left(\ell_{k}\right)} \leqslant C \ell_{n} / L L\left(\ell_{n}\right)$. For each $p \in(1,2)$, there exists a constant $K_{p}$ depending only on $p$ such that

$$
\left\|\sup _{n \geqslant 1} \frac{1}{\sqrt{\ell_{n} L L\left(\ell_{n}\right)}}\left|\sum_{\boldsymbol{i} \in \Lambda_{n}} X_{\boldsymbol{i}}\right|\right\|_{p} \leqslant K(p) C^{1 / p} \delta^{-1 / 2}\left\|\varepsilon_{\mathbf{0}}\right\|_{2} \sum_{\boldsymbol{j} \in \mathbb{Z}^{d}}\left|a_{\boldsymbol{j}}\right| .
$$

Note that the condition on $\ell_{n}$ is satisfied for instance when there exists positive constants $c_{1}$ and $c_{2}$ such that for each $n \geqslant 1, c_{1} 2^{n} \leqslant \ell_{n} \leqslant c_{2} 2^{n}$, or more generally, if $c_{1}\left\lfloor a^{n}\right\rfloor \leqslant \ell_{n} \leqslant c_{2}\left\lfloor a^{n}\right\rfloor$ for some $a>1$, where $\lfloor x\rfloor$ is the unique integer for which $\lfloor x\rfloor \leqslant x<\lfloor x\rfloor+1$.

We now present a result for arbitrary functionals of a linear random field, with the counterpart that the summation sets are assumed to be finite disjoint unions of rectangles. 
Theorem 1.3. Let $\left(X_{\boldsymbol{i}}\right)_{\boldsymbol{i} \in \mathbb{Z}^{d}}$ be a centered random field such that there exist an i.i.d. collection of random variables $\left\{\varepsilon_{\boldsymbol{u}}, \boldsymbol{u} \in \mathbb{Z}^{d}\right\}$ and a measurable function $f: \mathbb{R}^{\mathbb{Z}^{d}} \rightarrow \mathbb{R}$ such that $X_{\boldsymbol{i}}=$ $f\left(\left(\varepsilon_{\boldsymbol{i}-\boldsymbol{j}}\right)_{\boldsymbol{j} \in \mathbb{Z}^{d}}\right)$. Let $\left(\Gamma_{n}\right)_{n \geqslant 1}$ be a sequence of subsets of $\mathbb{Z}^{d}$ such that for each $n \geqslant 1, \Gamma_{n}$ is the disjoint union of $\Gamma_{n}(w), 1 \leqslant w \leqslant J_{n}$ and

$$
\Gamma_{n}(w)=\mathbb{Z}^{d} \cap \prod_{q=1}^{d}\left[\underline{n}_{q}(w, n), \bar{n}_{q}(w, n)\right], \bar{n}_{q}(w, n), \underline{n}_{q}(w, n) \in \mathbb{Z} .
$$

Assume that $\ell_{n}:=\operatorname{Card}\left(\Gamma_{n}\right)$ satisfies $\ell_{n+1} \geqslant \ell_{n} \geqslant \exp \left(n^{\delta}\right), \sum_{k=1}^{n} \sqrt{\ell_{k} / L L\left(\ell_{k}\right)} \leqslant C \ell_{n} / L L\left(\ell_{n}\right)$, where $C>0$ and $\delta>0$ are independent of $n$, and $\bar{n}_{q}(w, n)-\underline{n}_{q}(w, n) \geqslant 4$ for each $n \geqslant 1$, $1 \leqslant w \leqslant J_{n}, 1 \leqslant q \leqslant d$.

Then for all $1<p<2$, the following inequality holds:

$$
\left\|\sup _{n \geqslant 1} \frac{1}{\sqrt{\ell_{n} L L\left(\ell_{n}\right)}} \mid \sum_{\boldsymbol{i} \in \Gamma_{n}} X_{\boldsymbol{i}}\right\|\left\|_{p} \leqslant K(p, d, C, \delta) \sum_{j \geqslant 0}(j+1)^{d} L(j)^{1 / p}\right\| X_{\mathbf{0}, j} \|_{2},
$$

where $X_{\mathbf{0}, j}$ and $X_{\mathbf{0}, 0}$ are defined respectively by (1.2.3) and (1.2.4) and $K(p, d, C, \delta)$ depends on $p, d, C$ and $\delta$.

Notice that unlike the case of the supremum on rectangles addressed in Theorem 1.1, the $\mathbb{L}^{2}$-norm of the random variables $X_{\mathbf{0}, j}$ is involded instead of the norm $\|\cdot\|_{2, d-1}$. However, we need a condition on the size of the sets. Moreover, the weight term is $(j+1)^{d} L(j)^{1 / p}$ which is stronger than the term $(j+1)^{d / 2}$ appearing in Theorem 1.1 .

The terms $\left\|X_{\mathbf{0}, j}\right\|_{2, d-1}$ and $\left\|X_{\mathbf{0}, j}\right\|_{2}$ can be estimated by the so-called physical measure of dependence, introduced in [Wu05].

Definition 1.4. Let $\left(X_{\boldsymbol{i}}\right)_{\boldsymbol{i} \in \mathbb{Z}^{d}}$ be a strictly stationary random field which can be expressed as a functional of an i.i.d. random field, that is, there exist an i.i.d. random field $\left(\varepsilon_{\boldsymbol{u}}\right)_{\boldsymbol{u} \in \mathbb{Z}^{d}}$ and a measurable function $f: \mathbb{R}^{\mathbb{Z}^{d}} \rightarrow \mathbb{R}$ such that $X_{\boldsymbol{i}}=f\left(\left(\varepsilon_{\boldsymbol{i}-\boldsymbol{u}}\right)_{\boldsymbol{u} \in \mathbb{Z}^{d}}\right)$. Let $\varepsilon_{\mathbf{0}}^{\prime}$ be a random variable independent of $\left(\varepsilon_{\boldsymbol{u}}\right)_{\boldsymbol{u} \in \mathbb{Z}^{d}}$. Denote by $\varepsilon_{\boldsymbol{u}}^{*}$ the random variable $\varepsilon_{\boldsymbol{u}}$ if $\boldsymbol{u} \neq \mathbf{0}$ and $\varepsilon_{\mathbf{0}}^{\prime}$ if $\boldsymbol{u}=\mathbf{0}$.

For $r \geqslant 0$, we define the physical measure of dependence of $\left(X_{\boldsymbol{i}}\right)_{\boldsymbol{i} \in \mathbb{Z}^{d}}$ by

$$
\delta_{2, r}(\boldsymbol{i}):=\left\|f\left(\left(\varepsilon_{\boldsymbol{i}-\boldsymbol{u}}\right)_{\boldsymbol{u} \in \mathbb{Z}^{d}}\right)-f\left(\left(\varepsilon_{\boldsymbol{i}-\boldsymbol{u}}^{*}\right)_{\boldsymbol{u} \in \mathbb{Z}^{d}}\right)\right\|_{2, r}
$$

When the Orlicz norm $\|\cdot\|_{2, r}$ is replaced by the $\mathbb{L}^{p}$-norm, there are various examples of random fields where the measure of dependence is estimated (see Section 2 in [BD14] and [EVW13]). By using an appropriated version of Burkholder and Rosenthal's inequality in these spaces (see for instance Corollary 3.11), we can also estimate $\delta_{r}(\boldsymbol{i})$. This approach also allows to bound $\left\|X_{\mathbf{0}, j}\right\|_{2, d-1}$ by the coefficients $\delta_{d-1}(\boldsymbol{i})$, like in the proof of Corollary 1 in [Gir19]. This leads to the following result.

Corollary 1.5. Let $\left(X_{\boldsymbol{i}}\right)_{\boldsymbol{i} \in \mathbb{Z}^{d}}$ be a centered random field such that there exist an i.i.d. collection of random variables $\left\{\varepsilon_{\boldsymbol{u}}, \boldsymbol{u} \in \mathbb{Z}^{d}\right\}$ and a measurable function $f: \mathbb{R}^{\mathbb{Z}^{d}} \rightarrow \mathbb{R}$ such that $X_{\boldsymbol{i}}=$ $f\left(\left(\varepsilon_{\boldsymbol{i}-\boldsymbol{j}}\right)_{\boldsymbol{j} \in \mathbb{Z}^{d}}\right)$. For all $1<p<2$, the following inequality holds:

$$
\left\|\sup _{\boldsymbol{n} \in \mathbb{N}^{d}} \frac{1}{\sqrt{|\boldsymbol{n}| L L(|\boldsymbol{n}|)}} \mid \sum_{\mathbf{1} \preccurlyeq \boldsymbol{i} \preccurlyeq \boldsymbol{n}} X_{\boldsymbol{i}}\right\| \|_{p} \leqslant c_{p, d} \sum_{j \geqslant 0}(j+1)^{d / 2} \sqrt{\sum_{\boldsymbol{i} \in \mathbb{Z}^{d},\|\boldsymbol{i}\|_{\infty}=j} \delta_{2, d-1}(\boldsymbol{i})^{2}},
$$


where $c_{p, d}$ depends only on $p$ and $d$ and $\delta_{2, d-1}(\boldsymbol{i})$ is defined by (1.2.8).

Let us also mention the following consequence of Theorem 1.3.

Corollary 1.6. Let $\left(X_{\boldsymbol{i}}\right)_{\boldsymbol{i} \in \mathbb{Z}^{d}}$ be a centered random field such that there exist an i.i.d. collection of random variables $\left\{\varepsilon_{\boldsymbol{u}}, \boldsymbol{u} \in \mathbb{Z}^{d}\right\}$ and a measurable function $f: \mathbb{R}^{\mathbb{Z}^{d}} \rightarrow \mathbb{R}$ such that $X_{\boldsymbol{i}}=$ $f\left(\left(\varepsilon_{\boldsymbol{i}-\boldsymbol{j}}\right)_{\boldsymbol{j} \in \mathbb{Z}^{d}}\right)$. Let $\left(\Gamma_{n}\right)_{n \geqslant 1}$ be a sequence of subsets of $\mathbb{Z}^{d}$ such that for each $n \geqslant 1, \Gamma_{n}$ is the disjoint union of $\Gamma_{n}(w), 1 \leqslant w \leqslant J_{n}$ and

$$
\Gamma_{n}(w)=\mathbb{Z}^{d} \cap \prod_{q=1}^{d}\left[\underline{n}_{q}(w, n), \bar{n}_{q}(w, n)\right] .
$$

Assume that $\ell_{n}:=\operatorname{Card}\left(\Gamma_{n}\right)$ satisfies $\ell_{n+1} \geqslant \ell_{n} \geqslant \exp \left(n^{\delta}\right), \sum_{k=1}^{n} \sqrt{\ell_{k} / L L\left(\ell_{k}\right)} \leqslant C \ell_{n} / L L\left(\ell_{n}\right)$, where $C>0$ and $\delta>0$ are independent of $n$, and $\bar{n}_{q}(w, n)-\underline{n}_{q}(w, n) \geqslant 4$ for each $n \geqslant 1$, $1 \leqslant w \leqslant J_{n}, 1 \leqslant q \leqslant d$.

Then for all $1<p<2$, the following inequality holds:

$$
\begin{aligned}
\left\|\sup _{n \geqslant 1} \frac{1}{\sqrt{\ell_{n} L L\left(\ell_{n}\right)}}\left|\sum_{i \in \Gamma_{n}} X_{i}\right|\right\|_{p} & \leqslant(p, d, C, \delta) \sum_{j \geqslant 0}(j+1)^{d} L(j)^{1 / p} \sqrt{\sum_{i \in \mathbb{Z}^{d},\|\boldsymbol{i}\|_{\infty}=j} \delta_{2,0}(\boldsymbol{i})^{2}},
\end{aligned}
$$

where $\delta_{2,0}(\boldsymbol{i})$ is defined by (1.2.8).

\section{Applications}

In some particular cases of functionals of independent random fields, one can estimate $\left\|X_{\mathbf{0}, j}\right\|_{2, d-1}$ in terms of the parameters of the considered model. We will focus on the case of linear and Volterra random fields and functions of a Gaussian linear random field.

2.1. Functional of linear random fields. We say that the random fields $\left(X_{\boldsymbol{i}}\right)_{\boldsymbol{i} \in \mathbb{Z}^{d}}$ is a linear random field if there exists an i.i.d. centered random field $\left(\varepsilon_{\boldsymbol{i}}\right)_{\boldsymbol{i} \in \mathbb{Z}^{d}}$ of square integrable random variables and a family of real numbers $\left(a_{\boldsymbol{i}}\right)_{\boldsymbol{i} \in \mathbb{Z}^{d}}$ such that $\sum_{\boldsymbol{i} \in \mathbb{Z}^{d}} a_{\boldsymbol{i}}^{2}$ is finite and

$$
X_{j}=\sum_{i \in \mathbb{Z}^{d}} a_{i} \varepsilon_{j-i} \text { a.s.. }
$$

We will give a sufficient condition for the control of the maximal function of a random field which can be expressed as a Lipschitz function of a linear random field.

Corollary 2.1. Let $\left(X_{\boldsymbol{i}}\right)_{\boldsymbol{i} \in \mathbb{Z}^{d}}$ be a linear random field defined by (2.1.1), where $\left(\varepsilon_{\boldsymbol{i}}\right)_{\boldsymbol{i} \in \mathbb{Z}^{d}}$ is i.i.d.. Let $g: \mathbb{R} \rightarrow \mathbb{R}$ be a $\gamma$-Hölder continuous function where $0<\gamma \leqslant 1$ and assume that 
$\varepsilon_{\mathbf{0}} \in \mathbb{L}_{2 / \gamma, d-1}$ and $\mathbb{E}\left[g\left(X_{\mathbf{0}}\right)\right]=0$. Then for $1<p<2$,

$$
\begin{aligned}
\left\|\sup _{\boldsymbol{n} \in \mathbb{N}^{d}} \frac{1}{\sqrt{|\boldsymbol{n}| L L(|\boldsymbol{n}|)}}\left|\sum_{\mathbf{1} \preccurlyeq \boldsymbol{i} \preccurlyeq \boldsymbol{n}} g\left(X_{\boldsymbol{i}}\right)\right|\right\|_{p} \\
\qquad c_{p, d, \gamma} \sum_{j=0}^{+\infty}(j+1)^{d / 2}\left(\sum_{\boldsymbol{i} \in \mathbb{Z}^{d},\|\boldsymbol{i}\|_{\infty}=j}\left|a_{\boldsymbol{i}}\right|^{2 \gamma}\right)^{1 / 2}\left\|\varepsilon_{\mathbf{0}}\right\|_{2 \gamma, d-1},
\end{aligned}
$$

where $c_{p, d}$ depends only on $p, d$ and $\gamma$.

A similar result can be derived when the summation sets are finite disjoint unions of rectangles.

Corollary 2.2. Let $\left(X_{\boldsymbol{i}}\right)_{\boldsymbol{i} \in \mathbb{Z}^{d}}$ be a linear random field defined by (2.1.1), where $\left(\varepsilon_{\boldsymbol{i}}\right)_{\boldsymbol{i} \in \mathbb{Z}^{d}}$ is i.i.d.. Let $g: \mathbb{R} \rightarrow \mathbb{R}$ be a $\gamma$-Hölder continuous function where $0<\gamma \leqslant 1$ and assume that $\varepsilon_{\mathbf{0}} \in \mathbb{L}_{2 / \gamma, d-1}$ and $\mathbb{E}\left[g\left(X_{\mathbf{0}}\right)\right]=0$. Let $\left(\Gamma_{n}\right)_{n \geqslant 1}$ be a sequence of subsets of $\mathbb{Z}^{d}$ such that for each $n \geqslant 1, \Gamma_{n}$ is the disjoint union of $\Gamma_{n}(w), 1 \leqslant w \leqslant J_{n}$ and

$$
\Gamma_{n}(w)=\mathbb{Z}^{d} \cap \prod_{q=1}^{d}\left[\underline{n}_{q}(w, n), \bar{n}_{q}(w, n)\right] .
$$

Assume that $\ell_{n}:=\operatorname{Card}\left(\Gamma_{n}\right)$ satisfies $\ell_{n+1} \geqslant \ell_{n} \geqslant \exp \left(n^{\delta}\right), \sum_{k=1}^{n} \sqrt{\ell_{k} / L L\left(\ell_{k}\right)} \leqslant C \ell_{n} / L L\left(\ell_{n}\right)$, where $C>0$ and $\delta>0$ are independent of $n$, and $\bar{n}_{q}(w, n)-\underline{n}_{q}(w, n) \geqslant 4$ for each $n \geqslant 1$, $1 \leqslant w \leqslant J_{n}, 1 \leqslant q \leqslant d$.

Then for all $1<p<2$, the following inequality holds:

$$
\begin{aligned}
\left\|\sup _{n \geqslant 1} \frac{1}{\sqrt{\ell_{n} L L\left(\ell_{n}\right)}}\left|\sum_{\boldsymbol{i} \in \Gamma_{n}} X_{i}\right|\right\|_{p} \\
\leqslant K(p, d, C, \delta) \sum_{j \geqslant 0}(j+1)^{d} L(j)^{1 / p}\left(\sum_{i \in \mathbb{Z}^{d},\|\boldsymbol{i}\|_{\infty}=j}\left|a_{\boldsymbol{i}}\right|^{2 \gamma}\right)^{1 / 2}\left\|\varepsilon_{\mathbf{0}}\right\|_{2 \gamma, 0} .
\end{aligned}
$$

2.2. Functional of a Gaussian linear random field. Let $\left(\varepsilon_{\boldsymbol{i}}\right)_{\boldsymbol{i} \in \mathbb{Z}^{d}}$ be an i.i.d. random field where $\varepsilon_{\mathbf{0}}$ has a standard normal distribution. Let

$$
Y_{\boldsymbol{j}}:=\sum_{\boldsymbol{i} \in \mathbb{Z}^{d}} a_{\boldsymbol{i}} \varepsilon_{\boldsymbol{j}-\boldsymbol{i}}
$$

where $a_{\boldsymbol{i}}, \boldsymbol{i} \in \mathbb{Z}^{d}$, are real numbers such that $\sum_{\boldsymbol{i} \in \mathbb{Z}^{d}} a_{\boldsymbol{i}}^{2}=1$. Then $Y_{\boldsymbol{j}}=f\left(\left(\varepsilon_{\boldsymbol{j}-\boldsymbol{i}}\right)_{\boldsymbol{i} \in \mathbb{Z}^{d}}\right)$ where $f\left(\left(x_{\boldsymbol{u}}\right)_{\boldsymbol{u} \in \mathbb{Z}^{d}}\right)=\sum_{\boldsymbol{u} \in \mathbb{Z}^{d}} a_{\boldsymbol{u}} x_{\boldsymbol{u}}$ if $\sum_{\boldsymbol{u} \in \mathbb{Z}^{d}} a_{\boldsymbol{u}} x_{\boldsymbol{u}}$ converges (in the sense that $\lim _{m \rightarrow+\infty} \sum_{\substack{\boldsymbol{u} \in \mathbb{Z}_{\infty}^{d} \leqslant m \\ \|}} a_{\boldsymbol{u}} x_{\boldsymbol{u}}$ exists) and 0 otherwise. Let $\varepsilon_{\mathbf{0}}^{\prime}$ be a random variable having the same law as $\varepsilon_{\mathbf{0}}$ and independent of the random field $\left(\varepsilon_{\boldsymbol{i}}\right)_{\boldsymbol{i} \in \mathbb{Z}^{d}}$ and $Y_{\boldsymbol{j}}^{*}=f\left(\left(\varepsilon_{\boldsymbol{j}-\boldsymbol{i}}^{*}\right)_{\boldsymbol{i} \in \mathbb{Z}^{d}}\right)$, where $\varepsilon_{\boldsymbol{u}}^{*}=\varepsilon_{\boldsymbol{u}}$ if $\boldsymbol{u} \neq \mathbf{0}$ and $\varepsilon_{\mathbf{0}}^{*}=\varepsilon_{\mathbf{0}}^{\prime}$.

Let $\mathcal{H}_{0}=\mathbb{R}$ and let $\mathcal{H}_{1}$ be the first Wiener chaos defined as the closed subspace of $\mathbb{L}^{2}$ generated by the random variables $Y_{\boldsymbol{j}}$ and $Y_{\boldsymbol{j}}^{*}, \boldsymbol{j} \in \mathbb{Z}^{d}$. For $q \geqslant 2$, let $\mathcal{H}_{q}$ be the closed 
subspace of $\mathbb{L}^{2}$ generated by the random variables $H_{q}\left(Y_{\boldsymbol{j}}\right)$ and $H_{q}\left(Y_{\boldsymbol{j}}^{*}\right), \boldsymbol{j} \in \mathbb{Z}^{d}$, where $H_{q}$ is the $q$-th Hermite polynomial defined by

$$
H_{q}(x)=(-1)^{q} \exp \left(\frac{x^{2}}{2}\right) \frac{d^{q}}{d x^{q}} \exp \left(-\frac{x^{2}}{2}\right) .
$$

Given a function $f: \mathbb{R} \rightarrow \mathbb{R}$ such that $f\left(Y_{\mathbf{0}}\right) \in \mathbb{L}^{2}$ and $\mathbb{E}\left[f\left(Y_{\mathbf{0}}\right)\right]=0$, the following expansion holds:

$$
f\left(Y_{\boldsymbol{j}}\right)=\sum_{q=1}^{+\infty} c_{q}(f) H_{q}\left(Y_{\boldsymbol{j}}\right)
$$

where

$$
c_{q}(f)=\frac{1}{q !} \mathbb{E}\left[f\left(Y_{\mathbf{0}}\right) H_{q}\left(Y_{\mathbf{0}}\right)\right],
$$

provided that $\sum_{q=1}^{+\infty} q ! c_{q}(f)^{2}$ converges.

We are now in position to state the following consequence of Corollary 1.5 for functionals of a Gaussian linear random field.

Corollary 2.3. Let $\left(\varepsilon_{\boldsymbol{i}}\right)_{\boldsymbol{i} \in \mathbb{Z}^{d}}$ be an i.i.d. random field,

$$
Y_{j}:=\sum_{i \in \mathbb{Z}^{d}} a_{\boldsymbol{i}} \varepsilon_{j-i},
$$

where $a_{\boldsymbol{i}}, \boldsymbol{i} \in \mathbb{Z}^{d}$, are real numbers such that $\sum_{\boldsymbol{i} \in \mathbb{Z}^{d}} a_{\boldsymbol{i}}^{2}=1$. Let $f: \mathbb{R} \rightarrow \mathbb{R}$ be a function such that $\mathbb{E}\left[f\left(Y_{\mathbf{0}}\right)\right]=0$ and

$$
C(f):=\sum_{q=1}^{+\infty} \sqrt{q !} q^{d-\frac{1}{2}}\left|c_{q}(f)\right|<+\infty,
$$

where $c_{q}(f)$ is defined by (2.2.4). Then for all $1<p<2$,

$$
\begin{aligned}
\left\|\sup _{\boldsymbol{n} \in \mathbb{N}^{d}} \frac{1}{\sqrt{|\boldsymbol{n}| L L(|\boldsymbol{n}|)}}\left|\sum_{\mathbf{1} \boldsymbol{i} \preccurlyeq \boldsymbol{n}} f\left(Y_{\boldsymbol{i}}\right)\right|\right\|_{p} \\
\qquad c_{p, d} \sum_{j \geqslant 0}(j+1)^{d / 2}\left(\sum_{\boldsymbol{i} \in \mathbb{Z}^{d},\|\boldsymbol{i}\|_{\infty}=j} a_{i}^{2}\right)^{1 / 2} C(f),
\end{aligned}
$$

where $c_{p, d}$ depends only on $p$ and $d$.

Corollary 2.4. Let $\left(\varepsilon_{\boldsymbol{i}}\right)_{\boldsymbol{i} \in \mathbb{Z}^{d}}$ be an i.i.d. random field,

$$
Y_{j}:=\sum_{i \in \mathbb{Z}^{d}} a_{i} \varepsilon_{j-i}
$$

where $a_{\boldsymbol{i}}, \boldsymbol{i} \in \mathbb{Z}^{d}$, are real numbers such that $\sum_{\boldsymbol{i} \in \mathbb{Z}^{d}} a_{\boldsymbol{i}}^{2}=1$. Let $f: \mathbb{R} \rightarrow \mathbb{R}$ be a function such that $\mathbb{E}\left[f\left(Y_{\mathbf{0}}\right)\right]=0$ and

$$
C(f):=\sum_{q=1}^{+\infty} \sqrt{q !} q^{\frac{1}{2}}\left|c_{q}(f)\right|<+\infty,
$$


where $c_{q}(f)$ is defined by (2.2.4). Let $\left(\Gamma_{n}\right)_{n \geqslant 1}$ be a sequence of subsets of $\mathbb{Z}^{d}$ such that for each $n \geqslant 1, \Gamma_{n}$ is the disjoint union of $\Gamma_{n}(w), 1 \leqslant w \leqslant J_{n}$ and

$$
\Gamma_{n}(w)=\mathbb{Z}^{d} \cap \prod_{q=1}^{d}\left[\underline{n}_{q}(w, n), \bar{n}_{q}(w, n)\right] .
$$

Assume that $\ell_{n}:=\operatorname{Card}\left(\Gamma_{n}\right)$ satisfies $\ell_{n+1} \geqslant \ell_{n} \geqslant \exp \left(n^{\delta}\right), \sum_{k=1}^{n} \sqrt{\ell_{k} / L L\left(\ell_{k}\right)} \leqslant C \ell_{n} / L L\left(\ell_{n}\right)$, where $C>0$ and $\delta>0$ are independent of $n$, and $\bar{n}_{q}(w, n)-\underline{n}_{q}(w, n) \geqslant 4$ for each $n \geqslant 1$, $1 \leqslant w \leqslant J_{n}, 1 \leqslant q \leqslant d$.

Then for all $1<p<2$, the following inequality holds:

$$
\begin{aligned}
\left\|\sup _{n \geqslant 1} \frac{1}{\sqrt{\ell_{n} L L\left(\ell_{n}\right)}}\left|\sum_{\boldsymbol{i} \in \Gamma_{n}} f\left(Y_{\boldsymbol{i}}\right)\right|\right\|_{p} \\
\leqslant K(p, d, C, \delta) C(f) \sum_{j \geqslant 0}(j+1)^{d} L(j)^{1 / p}\left(\sum_{i \in \mathbb{Z}^{d},\|\boldsymbol{i}\|_{\infty}=j}\left|a_{\boldsymbol{i}}\right|^{2}\right)^{1 / 2} .
\end{aligned}
$$

2.3. Volterra processes. Volterra random fields of second order are defined in the following way. Let $\left(\varepsilon_{\boldsymbol{i}}\right)_{\boldsymbol{i} \in \mathbb{Z}^{d}}$ be an i.i.d. collection of centered random variables and $\left(a_{\boldsymbol{s}_{1}, \boldsymbol{s}_{\mathbf{2}}}\right)_{\boldsymbol{s}_{1}, \boldsymbol{s}_{2} \in \mathbb{Z}^{d}}$ be a family of real numbers such that $a_{\boldsymbol{s}_{1}, \boldsymbol{s}_{\mathbf{2}}}=0$ if $\boldsymbol{s}_{\mathbf{1}}=\boldsymbol{s}_{\mathbf{2}}$ and $\sum_{\boldsymbol{s}_{1}, \boldsymbol{s}_{\mathbf{2}} \in \mathbb{Z}^{d}} a_{\boldsymbol{s}_{1}, \boldsymbol{s}_{\mathbf{2}}}^{2}$ is finite. Define

$$
X_{j}:=\sum_{s_{1}, s_{2} \in \mathbb{Z}^{d}} a_{s_{1}, s_{2}} \varepsilon_{j-s_{1}} \varepsilon_{j-s_{2}} .
$$

One can bound the term $\left\|X_{\mathbf{0}, j}\right\|_{2, d-1}$, which leads to the following result.

Corollary 2.5. Let $\left(X_{\boldsymbol{i}}\right)_{\boldsymbol{i} \in \mathbb{Z}^{d}}$ be a Volterra random field defined by (2.3.1), where $\left(\varepsilon_{\boldsymbol{i}}\right)_{\boldsymbol{i} \in \mathbb{Z}^{d}}$ is i.i.d. and $\varepsilon_{\mathbf{0}} \in \mathbb{L}_{2, d-1}$. Then for $1<p<2$, the following inequality holds:

$$
\begin{aligned}
& \left\|\sup _{n \geqslant 1} \frac{1}{\sqrt{\ell_{n} L L\left(\ell_{n}\right)}}\left|\sum_{i \in \Gamma_{n}} X_{\boldsymbol{i}}\right|\right\|_{p} \\
& \leqslant c_{p, d} \sum_{j \geqslant 0}(j+1)^{d / 2}\left(\sum_{\left\|\boldsymbol{s}_{\mathbf{1}}\right\|_{\infty}=j} \sum_{\left\|\boldsymbol{s}_{\mathbf{2}}\right\|_{\infty} \leqslant j}\left(a_{\boldsymbol{s}_{1}, \boldsymbol{s}_{\mathbf{2}}}^{2}+a_{\boldsymbol{s}_{\mathbf{2}}, \boldsymbol{s}_{\mathbf{1}}}^{2}\right)\right)^{1 / 2}\left\|\varepsilon_{\mathbf{0}}\right\|_{2, d-1}^{2},
\end{aligned}
$$

where $c_{p, d}$ depends only on $p$ and $d$.

Corollary 2.6. Let $\left(X_{\boldsymbol{i}}\right)_{\boldsymbol{i} \in \mathbb{Z}^{d}}$ be a Volterra random field defined by (2.3.1), where $\left(\varepsilon_{\boldsymbol{i}}\right)_{\boldsymbol{i} \in \mathbb{Z}^{d}}$ is i.i.d. and $\varepsilon_{\mathbf{0}} \in \mathbb{L}^{2}$. Then for $1<p<2$, the following inequality holds:

$$
\begin{aligned}
& \left\|\sup _{\boldsymbol{n} \in \mathbb{N}^{d}} \frac{1}{\sqrt{|\boldsymbol{n}| L L(|\boldsymbol{n}|)}}\left|\sum_{\mathbf{1} \preccurlyeq i \preccurlyeq \boldsymbol{n}} X_{\boldsymbol{i}}\right|\right\|_{p} \\
& \leqslant K(p, d, C, \delta) \sum_{j \geqslant 0}(j+1)^{d} L(j)^{1 / p}\left(\sum_{\left\|\boldsymbol{s}_{1}\right\|_{\infty}=j} \sum_{\left\|\boldsymbol{s}_{\mathbf{2}}\right\|_{\infty} \leqslant j}\left(a_{\boldsymbol{s}_{1}, \boldsymbol{s}_{\mathbf{2}}}^{2}+a_{\boldsymbol{s}_{2}, \boldsymbol{s}_{1}}^{2}\right)\right)^{1 / 2}\left\|\varepsilon_{\mathbf{0}}\right\|_{2,0}^{2} .
\end{aligned}
$$

\section{Proofs}

\subsection{Tools for the proofs.}


3.1.1. Global ideas of proofs. Let us explain the main steps in the proof of the results. For Theorem 1.1, we proceed as follows.

(1) As a first step, we prove Theorem 1.1 in the i.i.d. case. To this aim, we show that the maximal function involved in Theorem 1.1 can be replaced by another one where the supremum is taken only over elements of $\mathbb{N}^{d}$ having dyadic coordinates. Then we apply an appropriated exponential inequality in order to control the moments of this new maximal function. This gives Theorem 3.15.

(2) In order to prove Theorem 1.1, we bound the maximal function by a series of maximal functions associated to an i.i.d. random field. The contribution of these maximal functions can be estimated by using Theorem 3.15.

For Theorem 1.2, we reduce the problem to the i.i.d. case. We use a truncation argument and the deviation inequality given in Proposition 3.4.

Theorem 1.3 rests on Theorem 1.2 and a bound on the maximal function by maximal function of an i.i.d. random field. However, we need to reduce the summation on subsets of the union of rectangles and check that these sets satisfy the assumptions of Theorem 1.2 with the explicit constants.

3.1.2. Weak $\mathbb{L}^{p}$-spaces. The results of the paper provide a control of the $\mathbb{L}^{p}$ norm of a maximal function. However, it will sometimes be more convenient to work directly with tails. To this aim, we will consider weak $\mathbb{L}^{p}$-spaces.

Definition 3.1. Let $p>1$. The weak $\mathbb{L}^{p}$-space, denoted by $\mathbb{L}^{p, w}$, is the space of random variables $X$ such that $\sup _{t>0} t^{p} \mathbb{P}\{|X|>t\}$ is finite.

These spaces can be endowed with a norm.

Lemma 3.2. Let $1<p<2$. Define the following norm on $\mathbb{L}^{p, w}$

$$
\|X\|_{p, w}:=\sup \left\{\mathbb{P}(A)^{1 / p-1} \mathbb{E}\left[|X| \mathbf{1}_{A}\right]\right\} .
$$

For all random variable $X \in \mathbb{L}^{p, w}$, the following inequality holds:

$$
c_{p}\|X\|_{p, w} \leqslant\left(\sup _{t>0} t^{p} \mathbb{P}\{|X|>t\}\right)^{1 / p} \leqslant C_{p}\|X\|_{p, w} \leqslant C_{p}\|X\|_{p}
$$

where $c_{p}$ and $C_{p}$ depend only on $p$.

3.1.3. Deviation inequalities. The following deviation inequality is consequence of Theorem 2.1 in [BT08], which states that for a square integrable martingale differences sequence $\left(d_{j}\right)_{j=1}^{n}$ with respect to a filtration $\left(\mathcal{F}_{j}\right)_{j \geqslant 0}$,

$$
\mathbb{P}\left(\left\{\left|\sum_{j=1}^{n} d_{j}\right|>x\right\} \cap\left\{\sum_{j=1}^{n}\left(d_{j}^{2}+\mathbb{E}\left[d_{j}^{2} \mid \mathcal{F}_{j-1}\right]\right) \leqslant y\right\}\right) \leqslant 2 \exp \left(-\frac{x^{2}}{2 y}\right) .
$$

Proposition 3.3. Let $\left(d_{j}\right)_{j \geqslant 1}$ be a square integrable independent sequence. Then for all positive numbers $x$ and $y$, the following inequality holds:

$$
\mathbb{P}\left(\left\{\left|\sum_{j=1}^{n} d_{j}\right|>x\right\} \cap\left\{\sum_{j=1}^{n} d_{j}^{2} \leqslant y\right\}\right) \leqslant 2 \exp \left(-\frac{x^{2}}{2\left(y+V^{2}\right)}\right),
$$


where $V^{2}=\sum_{j=1}^{n} \mathbb{E}\left[d_{j}^{2}\right]$.

This follows from an application of (3.1.3) to $\mathcal{F}_{i}=\sigma\left(d_{j}, 1 \leqslant j \leqslant i\right)$ and $y$ replaced by $y+V^{2}$.

The following inequality will be needed in the proof of Theorem 1.2; this is a version of Proposition A.1 in [DGM10], stated in this paper for martingale differences but presented here for independent sequences.

Proposition 3.4. Let $\left(d_{j}\right)_{j \geqslant 1}$ be an independent centered sequence such that there exists a $c>0$ for which $\left|d_{j}\right| \leqslant c$ almost surely. Let $n \geqslant 1$ be an integer and let $y$ be a real number such that $y \geqslant \sum_{i=1}^{n} \mathbb{E}\left[d_{i}^{2}\right]$. For all $x>0$, the inequality

$$
\mathbb{P}\left\{\left|\sum_{i=1}^{n} d_{i}\right|>x\right\} \leqslant 2 \exp \left(-\frac{y}{c^{2}} h\left(\frac{x c}{y}\right)\right)
$$

takes place, where $h(u)=(1+u) \ln (1+u)-u$.

Lemma 3.5. Assume that $X$ and $Y$ are two non-negative random variables such that for each positive $x$, we have

$$
x \mathbb{P}\{X>x\} \leqslant \mathbb{E}[Y \mathbf{1}\{X \geqslant x\}] .
$$

Then for each $t$, the following inequality holds:

$$
\mathbb{P}\{X>2 t\} \leqslant \int_{1}^{+\infty} \mathbb{P}\{Y>s t\} \mathrm{d} s .
$$

Lemma 3.6. Let $X$ be an integrable non-negative random variable and let $\mathcal{G}$ be a sub- $\sigma$-algebra of $\mathcal{F}$. For all real number $y$, the following inequality holds:

$$
\int_{1 / 2}^{+\infty} \mathbb{P}\{\mathbb{E}[X \mid \mathcal{G}]>y u\} \mathrm{d} u \leqslant 2 \int_{1 / 2}^{+\infty} \mathbb{P}\{X>y u\} \mathrm{d} u .
$$

Proof. Replacing $X$ by $X / y$, there is no loss of generality by assuming that $y=1$. For any non-negative random variable $Z$, we have

$$
\mathbb{E}[Z \mathbf{1}\{Z>1 / 2\}]=\int_{1 / 2}^{\infty} \mathbb{P}\{Z>t\} \mathrm{d} t+\frac{1}{2} \mathbb{P}\{Z>1 / 2\}
$$

Therefore,

$$
\int_{1 / 2}^{+\infty} \mathbb{P}\{\mathbb{E}[X \mid \mathcal{G}]>u\} \mathrm{d} u \leqslant \mathbb{E}[\mathbb{E}[X \mid \mathcal{G}] \mathbf{1}\{\mathbb{E}[X \mid \mathcal{G}]>1 / 2\}]
$$

and by definition of condition expectation, we get

$$
\int_{1 / 2}^{+\infty} \mathbb{P}\{\mathbb{E}[X \mid \mathcal{G}]>u\} \mathrm{d} u \leqslant \mathbb{E}[X \mathbf{1}\{\mathbb{E}[X \mid \mathcal{G}]>1 / 2\}]
$$

The last expectation can be written as

$$
\int_{0}^{+\infty} \mathbb{P}(\{X>t\} \cap\{\mathbb{E}[X \mid \mathcal{G}]>1 / 2\}) \mathrm{d} t \leqslant \frac{1}{2} \mathbb{P}\{\mathbb{E}[X \mid \mathcal{G}]>1 / 2\}+\int_{1 / 2}^{+\infty} \mathbb{P}\{X>t\} \mathrm{d} t .
$$

The first term of the right hand side does not exceed $1 / 2 \int_{1 / 2}^{+\infty} \mathbb{P}\{\mathbb{E}[X \mid \mathcal{G}]>u\}$ d $u$ hence

$$
\mathbb{E}[X \mathbf{1}\{\mathbb{E}[X \mid \mathcal{G}]>1 / 2\}] \leqslant \frac{1}{2} \int_{1 / 2}^{+\infty} \mathbb{P}\{\mathbb{E}[X \mid \mathcal{G}]>u\} \mathrm{d} u
$$

We finish the proof by combining (3.1.11) with (3.1.12). 
Proposition 3.7. Let $\left(Y_{\boldsymbol{i}}\right)_{\boldsymbol{i} \in \mathbb{Z}^{d}}$ be a strictly stationary random field such that each $Y_{\boldsymbol{i}}$ is a non-negative random variable. Then for all positive $y$,

$$
\mathbb{P}\left\{\sup _{\boldsymbol{n} \in \mathbb{N}^{d}} \frac{1}{|\boldsymbol{n}|} \sum_{\mathbf{1} \preccurlyeq \boldsymbol{i} \preccurlyeq \boldsymbol{n}} Y_{\boldsymbol{i}}>y\right\} \leqslant \int_{1}^{+\infty} \mathbb{P}\left\{Y_{\mathbf{1}}>y u 2^{-d}\right\}(\log u)^{d-1} \mathrm{~d} u .
$$

Proof. The proof is done by induction on the dimension. For $d=1$, this follows from a combination of the maximal ergodic theorem with Lemma 3.5. Suppose now that for some $d \geqslant 2$, Proposition 3.7 holds for all $(d-1)$-dimensional random fields and let $\left(Y_{\boldsymbol{i}}\right)_{\boldsymbol{i} \in \mathbb{Z}^{d}}$ be a strictly stationary random field such that each $Y_{\boldsymbol{i}}$ is a non-negative random variable. For $i_{1}, \ldots, i_{d-1} \in \mathbb{N}$, define

$$
\widetilde{Y}_{i_{1}, \ldots, i_{d-1}}:=\sup _{n \geqslant 1} \frac{1}{n} \sum_{i=1}^{n} Y_{i_{1}, i_{2}, \ldots, i_{d-1}, i}
$$

Then

$$
\sup _{\boldsymbol{n} \in \mathbb{N}^{d}} \frac{1}{|\boldsymbol{n}|} \sum_{\mathbf{1} \preccurlyeq \boldsymbol{i} \preccurlyeq \boldsymbol{n}} Y_{\boldsymbol{i}} \leqslant \sup _{\boldsymbol{n} \in \mathbb{N}^{d-1}} \frac{1}{|\boldsymbol{n}|} \sum_{\mathbf{1} \preccurlyeq \boldsymbol{i} \preccurlyeq \boldsymbol{n}} \tilde{Y}_{\boldsymbol{i}} .
$$

Applying the induction hypothesis to the strictly stationary random field $\left(\widetilde{Y}_{i}\right)_{i \in \mathbb{Z}^{d-1}}$, we get

$$
\mathbb{P}\left\{\sup _{\boldsymbol{n} \succcurlyeq \mathbf{1}} \frac{1}{|\boldsymbol{n}|} \sum_{\mathbf{1} \preccurlyeq \boldsymbol{i} \preccurlyeq \boldsymbol{n}} Y_{\boldsymbol{i}}>y\right\} \leqslant \int_{1}^{+\infty} \mathbb{P}\left\{\widetilde{Y_{\mathbf{0}}}>y u 2^{1-d}\right\}(\log u)^{d-2} \mathrm{~d} u .
$$

Applying the one dimensional case to the strictly stationary sequence $\left(Y_{0, \ldots, 0, i}\right)_{i \geqslant 1}$ gives

$$
\mathbb{P}\left\{\sup _{\boldsymbol{n} \in \mathbb{N}^{d}} \frac{1}{|\boldsymbol{n}|} \sum_{\mathbf{1} \preccurlyeq \boldsymbol{i} \preccurlyeq \boldsymbol{n}} Y_{\boldsymbol{i}}>y\right\} \leqslant \int_{1}^{+\infty} \int_{1}^{+\infty} \mathbb{P}\left\{Y_{\mathbf{0}}>y u v 2^{-d}\right\}(\log u)^{d-2} \mathrm{~d} v \mathrm{~d} u .
$$

and rearranging the integrals ends the proof.

\subsubsection{Facts on Orlicz spaces.}

Lemma 3.8. Let $p \geqslant 1$ and $r \geqslant 0$. Let $\varphi:=\varphi_{p, q}$ and let $a>0$ be a constant. There exists a constant $c$ depending only on $a, p$ and $q$ such that for all random variable $X$,

$$
\|X\|_{\varphi} \leqslant c\|X\|_{a \varphi} .
$$

Proof. By homogeneity, it suffices to prove that for all random variable $X$ such that $\|X\|_{a \varphi}=1$, the equality $\|X\|_{\varphi} \leqslant c$ holds for some $c$ depending only on $a, p$ and $q$. Let $X$ be a random variable such that $\|X\|_{a \varphi}=1$. Then we know that $\mathbb{E}[a \varphi(X)]=1$. Since there exists a constant $K$ such that $\varphi(u v) \leqslant K \varphi(u) \varphi(v)$, we derive that

$$
\mathbb{E}[\varphi(X / c)] \leqslant \frac{1}{a} a K \mathbb{E}[\varphi(X) \varphi(1 / c)]=K \frac{1}{a} \varphi(1 / b) .
$$

We choose $c$ such that $K \frac{1}{a} \varphi(1 / c) \leqslant 1$; for such a $c$, inequality $\|X\|_{\varphi} \leqslant c$ holds, which ends the proof.

Lemma 3.9. Let $r \geqslant 0$. There exists a constant $c_{r}$ such that for any random variable $X$,

$$
\begin{gathered}
\left\|X^{2}\right\|_{1, r} \leqslant c_{r}\|X\|_{2, r}^{2} ; \\
\left\|X^{1 / 2}\right\|_{2, r} \leqslant c_{r}\|X\|_{1, r}^{1 / 2} .
\end{gathered}
$$


Proof. We use the fact that there exists a constant $c$ depending only on $r$ such that for all positive $x$,

$$
c^{-1} \varphi_{1, r}\left(x^{2}\right) \leqslant \varphi_{2, r}(x) \leqslant c \varphi_{1, r}\left(x^{2}\right)
$$

Let us prove (3.1.19). By homogeneity, we assume that $\|X\|_{2, r}=1$. Then

$$
\mathbb{E}\left[\varphi_{1, r}\left(X^{2}\right)\right] \leqslant c \mathbb{E}\left[\varphi_{2, r}(X)\right] \leqslant c,
$$

which shows that $\left\|X^{2}\right\|_{\varphi_{2, r} / c} \leqslant 1$ and we conclude by applying Lemma 3.8.

The proof of (3.1.20) follows exactly the same lines.

A tool will be the following version of Burkholder's inequality, which is a consequence of the combination of Lemmas 3.1 and 6.1 in [Bur73] (which is valid, since $\varphi_{2, r}$ satisfies $\left.\sup _{t>0} \varphi_{2, r}(2 t) / \varphi_{2, r}(t)<+\infty\right)$.

Proposition 3.10. Let $r \geqslant 0$ be a real number and let $\left(d_{k}\right)_{k=1}^{n}$ be a martingale differences sequence with respect to the filtration $\left(\mathcal{F}_{k}\right)_{k=0}^{n}$. The following inequality holds

$$
\left\|\sum_{k=1}^{n} d_{k}\right\|_{2, r} \leqslant C_{r}\left\|\max _{1 \leqslant k \leqslant n}\left|d_{k}\right|\right\|_{2, r}+C_{r}\left\|\left(\sum_{k=1}^{n} \mathbb{E}\left[d_{k}^{2} \mid \mathcal{F}_{k-1}\right]\right)^{1 / 2}\right\|_{2, r} .
$$

Using Lemma 3.8, we derive that

$$
\begin{aligned}
\left\|\left(\sum_{k=1}^{n} \mathbb{E}\left[d_{k}^{2} \mid \mathcal{F}_{k-1}\right]\right)^{1 / 2}\right\|_{2, r} \leqslant c_{r}\left\|\sum_{k=1}^{n} \mathbb{E}\left[d_{k}^{2} \mid \mathcal{F}_{k-1}\right]\right\|_{1, r}^{1 / 2} \\
\leqslant c_{r}\left(\sum_{k=1}^{n}\left\|\mathbb{E}\left[d_{k}^{2} \mid \mathcal{F}_{k-1}\right]\right\|_{1, r}\right)^{1 / 2}
\end{aligned}
$$

and the following corollary.

Corollary 3.11. Let $r \geqslant 0$ be a real number and let $\left(d_{k}\right)_{k=1}^{n}$ be a martingale differences sequence with respect to the filtration $\left(\mathcal{F}_{k}\right)_{k=0}^{n}$. The following inequalities hold

$$
\begin{gathered}
\left\|\sum_{k=1}^{n} d_{k}\right\|_{2, r} \leqslant C_{r}\left\|\max _{1 \leqslant k \leqslant n}\left|d_{k}\right|\right\|_{2, r}+C_{r}\left(\sum_{k=1}^{n}\left\|\mathbb{E}\left[d_{k}^{2} \mid \mathcal{F}_{k-1}\right]\right\|_{1, r}\right)^{1 / 2} ; \\
\left\|\sum_{k=1}^{n} d_{k}\right\|_{2, r}^{2} \leqslant K_{r} \sum_{k=1}^{n}\left\|d_{k}\right\|_{2, r}^{2},
\end{gathered}
$$

where $C_{r}$ and $K_{r}$ depend only on $r$.

Observe also that

$$
\left\|X^{2}\right\|_{1, r} \leqslant c_{r}\|X\|_{2, r}^{2} .
$$

Lemma 3.12. For all $p>1$ and $r \geqslant 0$, there exists a constant $c_{p, r}$ such that if $X$ and $Y$ are two non-negative random variables satisfying for each positive $x$,

$$
x \mathbb{P}\{X>x\} \leqslant \mathbb{E}[Y \mathbf{1}\{X \geqslant x\}],
$$

then $\|X\|_{p, r} \leqslant c_{p, r}\|Y\|_{p, r}$. 
Proof. This follows from a rewritting of $\mathbb{E}\left[\varphi_{p, r}(X)\right]$ using tails of $X$, that is,

$$
\mathbb{E}\left[\varphi_{p, r}(X)\right]=\int_{0}^{+\infty} \varphi_{p, r}^{\prime}(t) \mathbb{P}\{X>t\} \mathrm{d} t .
$$

Since $\varphi_{p, r}^{\prime}(t) \leqslant c t^{p-1}(1+\log (1+t))^{r} \leqslant C \varphi_{p, r}^{\prime}(t)$, we derive by Lemma 3.5 that

$$
\begin{aligned}
& \mathbb{E}\left[\varphi_{p, r}(X)\right] \leqslant c \int_{0}^{+\infty} t^{p-1}(1+\log (1+t))^{r} \int_{1}^{+\infty} \mathbb{P}\{Y>x t / 2\} \mathrm{d} x \mathrm{~d} t \\
& \leqslant c C \int_{1}^{+\infty} \mathbb{E}\left[\varphi_{p, r}(2 Y / x) \mathbf{1}\{2 Y>x\}\right] \mathrm{d} x .
\end{aligned}
$$

Since $\varphi_{p, r}(u v) \leqslant K \varphi_{p, r}(u) \varphi_{p, r}(v)$ and the integral $\int_{1}^{+\infty} \varphi_{p, r}(1 / x) \mathrm{d} x$ converges, we proved that $\mathbb{E}\left[\varphi_{p, r}(X)\right] \leqslant c_{p, r} \mathbb{E}\left[\varphi_{p, r}(Y)\right]$ and we conclude using Lemma 3.8.

3.2. Reduction to dyadics. Let $d$ be a fixed integer and for $0 \leqslant i \leqslant d-1$ define by $\mathbb{N}_{i}$ the elements of $\mathbb{N}^{d}$ whose coordinates $i+1, \ldots, d$ are dyadic numbers. More formally,

$$
\mathbb{N}_{i}:=\left\{\boldsymbol{n} \in \mathbb{N}^{d} \mid \text { for all } i+1 \leqslant j \leqslant d, \exists k_{j} \in \mathbb{N} \cup\{0\} \text { such that } n_{j}=2^{k_{j}}\right\} .
$$

We also define $\mathbb{N}_{d}$ as $\mathbb{N}^{d}$. Notice that $\mathbb{N}_{0}$ is the set of all the elements of $\mathbb{N}^{d}$ such that all the coordinates are powers of 2 of a non-negative integer. The goal of this subsection is to show that it suffices to prove Theorem 1.1 where the supremum over $\mathbb{N}^{d}$ is replaced by the corresponding one over $\mathbb{N}_{0}$.

Proposition 3.13. Let $\left(X_{i}\right)_{i \in \mathbb{Z}^{d}}$ be an i.i.d. centered random field. Then for all $1<p<2$, the following inequality holds

$$
\left\|\sup _{\boldsymbol{n} \in \mathbb{N}^{d}} \frac{1}{\sqrt{|\boldsymbol{n}| L L(|\boldsymbol{n}|)}}\left|\sum_{\mathbf{1} \preccurlyeq \boldsymbol{i} \preccurlyeq \boldsymbol{n}} X_{\boldsymbol{i}}\right|\right\|_{p} \leqslant c_{p, d}\left\|\sup _{\boldsymbol{n} \in \mathbb{N}_{0}} \frac{1}{\sqrt{|\boldsymbol{n}| L L(|\boldsymbol{n}|)}}\left|\sum_{\mathbf{r} \preccurlyeq \boldsymbol{i} \preccurlyeq \boldsymbol{n}} X_{\boldsymbol{i}}\right|\right\|_{p},
$$

where $c_{p, d}$ depends only on $d$.

Lemma 3.14. Let $\left(a_{\boldsymbol{n}}\right)_{\boldsymbol{n} \in \mathbb{N}^{d}}$ be a family of positive numbers such that $a_{\boldsymbol{n}} \leqslant a_{\boldsymbol{n}^{\prime}}$ if $\boldsymbol{n} \preccurlyeq \boldsymbol{n}^{\prime}$ and

$$
c:=\sup _{\boldsymbol{n} \in \mathbb{N}^{d}} \max _{1 \leqslant i \leqslant d} \frac{a_{\boldsymbol{n}+n_{i} \boldsymbol{e}_{\boldsymbol{i}}}}{a_{\boldsymbol{n}}}<+\infty .
$$

Assume that $\left(X_{\boldsymbol{i}}\right)_{\boldsymbol{i} \in \mathbb{Z}^{d}}$ is independent and centered. Then for any real number number $x$ and any $i \in\{1, \ldots, d\}$,

$$
\mathbb{P}\left\{M_{i}>x\right\} \leqslant \int_{1}^{+\infty} \mathbb{P}\left\{M_{i-1}>\frac{u x}{2 c}\right\} \mathrm{d} u,
$$

where

$$
M_{i}=\sup _{\boldsymbol{n} \in \mathbb{N}_{i}} \frac{1}{\sqrt{|\boldsymbol{n}| L L(|\boldsymbol{n}|)}}\left|\sum_{\mathbf{1} \preccurlyeq \boldsymbol{i} \preccurlyeq \boldsymbol{n}} X_{\boldsymbol{i}}\right| .
$$

Proof. Let $0 \leqslant i \leqslant d-1$. Define the random variables

$$
\begin{gathered}
Y_{N}:=\frac{1}{a_{n_{1}, \ldots, n_{i-1}, N, n_{i+1}, \ldots, n_{d}}} \sup _{n_{1}, \ldots, n_{i-1} n_{i+1}, \ldots, n_{d}} \sup _{n_{1}, \ldots, n_{i-1}, N, n_{i+1}, \ldots, n_{d}} \mid, \\
Y_{N}^{\prime}:=\frac{a_{n_{1}, \ldots, n_{i-1}, N, n_{i+1}, \ldots, n_{d}}}{a_{n_{1}, \ldots, n_{i-1}, 2^{n+1}, n_{i+1}, \ldots, n_{d}}} Y_{N}, \quad 2^{n}+1 \leqslant N \leqslant 2^{n+1} .
\end{gathered}
$$


and the following events

$$
\begin{aligned}
A_{N} & :=\left\{Y_{N}>x\right\}, B_{0}=\emptyset, B_{N}:=A_{N} \backslash \bigcup_{i=0}^{N-1} A_{i}, \\
C_{N, n} & := \begin{cases}\bigcup_{i=2^{n}+1}^{N} B_{i}, & \text { if } 2^{n}+1 \leqslant N \leqslant 2^{n+1} ; \\
\emptyset, & \text { if } N \leqslant 2^{n} \text { or } N>2^{n+1} .\end{cases}
\end{aligned}
$$

In this way, the set $\left\{M_{i}>x\right\}$ can be expressed as the disjoint union $\bigcup_{N \geqslant 1} B_{N}$ hence

$$
\mathbb{P}\left\{M_{i}>x\right\} \leqslant \sum_{N \geqslant 1} \mathbb{P}\left(B_{N}\right)=\sum_{n=0}^{+\infty} \sum_{N=2^{n}+1}^{2^{n+1}} \mathbb{P}\left(B_{N}\right) .
$$

Since $x \mathbf{1}\left(B_{N}\right) \leqslant Y_{N} \mathbf{1}\left(B_{N}\right)$, we infer that

$$
x \mathbb{P}\left\{M_{i}>x\right\} \leqslant \sum_{n=0}^{+\infty} \sum_{N=2^{n}+1}^{2^{n+1}} \mathbb{E}\left[Y_{N} \mathbf{1}\left(B_{N}\right)\right] .
$$

By definition of $c$ in $(3.2 .3)$, we get that

$$
x \mathbb{P}\left\{M_{i}>x\right\} \leqslant c \sum_{n=0}^{+\infty} \sum_{N=2^{n}+1}^{2^{n+1}} \mathbb{E}\left[Y_{N}^{\prime} \mathbf{1}\left(B_{N}\right)\right] .
$$

Let $n \geqslant 0$ be fixed. Since $\mathbf{1}\left(B_{N}\right)=\mathbf{1}\left(C_{N, n}\right)-\mathbf{1}\left(C_{N-1, n}\right)$ for all $n$ such that $2^{n}+1 \leqslant N \leqslant 2^{n+1}$,

$$
\begin{aligned}
\sum_{N=2^{n}+1}^{2^{n+1}} \mathbb{E}\left[Y_{N}^{\prime} \mathbf{1}\left(B_{N}\right)\right] & =\sum_{N=2^{n}+1}^{2^{n+1}} \mathbb{E}\left[Y_{N}^{\prime}\left(\mathbf{1}\left(C_{N, n}\right)-\mathbf{1}\left(C_{N-1, n}\right)\right)\right] \\
& =\mathbb{E}\left[\sum_{N=2^{n}+1}^{2^{n+1}} Y_{N}^{\prime} \mathbf{1}\left(C_{N, n}\right)-\sum_{N=2^{n}}^{2^{n+1}-1} Y_{N+1}^{\prime} \mathbf{1}\left(C_{N, n}\right)\right] \\
& \leqslant \mathbb{E}\left[Y_{2^{n+1}}^{\prime} \mathbf{1}\left(C_{2^{n+1}, n}\right)\right]+\mathbb{E}\left[\sum_{N=2^{n}+1}^{2^{n+1}-1}\left(Y_{N}^{\prime}-Y_{N+1}^{\prime}\right) \mathbf{1}\left(C_{N, n}\right)\right] .
\end{aligned}
$$

The set $\mathbf{1}\left(C_{N, n}\right)$ is measurable with respect to the $\sigma$-algebra $\mathcal{G}_{N}$ generated by the random variables $\varepsilon_{\boldsymbol{u}}, \boldsymbol{u} \in \mathbb{Z}^{d}, u_{i} \leqslant N$, and by independence of $\left(X_{\boldsymbol{i}}\right)_{\boldsymbol{i} \in \mathbb{Z}^{d}}$ the random variable $\mathbb{E}\left[Y_{N+1}^{\prime}-Y_{N}^{\prime} \mid \mathcal{G}_{N}\right]$ is non-negative and consequently,

$$
\begin{aligned}
\mathbb{E}\left[\left(Y_{N}^{\prime}-Y_{N+1}^{\prime}\right) \mathbf{1}\left(C_{N, n}\right)\right] & =\mathbb{E}\left[\mathbb{E}\left[\left(Y_{N}^{\prime}-Y_{N+1}^{\prime}\right) \mathbf{1}\left(C_{N, n}\right) \mid \mathcal{G}_{N}\right]\right] \\
& =\mathbb{E}\left[\mathbf{1}\left(C_{N, n}\right) \mathbb{E}\left[Y_{N}^{\prime}-Y_{N+1}^{\prime} \mid \mathcal{G}_{N}\right]\right] \leqslant 0
\end{aligned}
$$

from which it follows that

$$
\sum_{N=2^{n}+1}^{2^{n+1}} \mathbb{E}\left[Y_{N}^{\prime} \mathbf{1}\left(B_{N}\right)\right] \leqslant \mathbb{E}\left[Y_{2^{n+1}}^{\prime} \mathbf{1}\left(C_{N, n}\right)\right] .
$$

The latter inequality combined with (3.2.12) allows to deduce that

$$
x \mathbb{P}\left\{M_{i}>x\right\} \leqslant c \sum_{n=0}^{+\infty} \mathbb{E}\left[Y_{2^{n+1}}^{\prime} \mathbf{1}\left(C_{2^{n+1}, n}\right)\right] .
$$


Observe that for all $n \geqslant 0$, the random variable $Y_{2^{n+1}}^{\prime}$ is bounded by $M_{i-1}$. Combining this with the definition of $C_{N, n}$ given by (3.2.9), we derive that

$$
x \mathbb{P}\left\{M_{i}>x\right\} \leqslant c \sum_{n=0}^{+\infty} \mathbb{E}\left[M_{i-1} \mathbf{1}\left(\bigcup_{k=2^{n}+1}^{2^{n+1}} B_{k}\right)\right] .
$$

Since the family $\left\{B_{k}, k \geqslant 1\right\}$ is pairwise disjoint, so is the family $\left\{\bigcup_{k=2^{n}+1}^{2^{n+1}} B_{k}, n \geqslant 0\right\}$. Therefore, using again the fact that $\left\{M_{i}>x\right\}$ can be expressed as the disjoint union $\bigcup_{N \geqslant 1} B_{N}$, we establish the inequality

$$
x \mathbb{P}\left\{M_{i}>x\right\} \leqslant c \mathbb{E}\left[M_{i-1} \mathbf{1}\left\{M_{i}>x\right\}\right] .
$$

We estimate the right hand side of the previous inequality in the following way:

$$
\begin{aligned}
\mathbb{E}\left[M_{i-1} \mathbf{1}\left\{M_{i}>x\right\}\right] & =\int_{0}^{+\infty} \mathbb{P}\left(\left\{M_{i}>x\right\} \cap\left\{M_{i-1}>t\right\}\right) \mathrm{d} t \\
& \leqslant \int_{0}^{x /(2 c)} \mathbb{P}\left\{M_{i}>x\right\} \mathrm{d} t+\int_{x /(2 c)}^{+\infty} \mathbb{P}\left\{M_{i-1}>t\right\} \mathrm{d} t \\
& =\frac{x}{2 c} \mathbb{P}\left\{M_{i}>x\right\}+\frac{x}{2 c} \int_{1}^{+\infty} \mathbb{P}\left\{M_{i-1}>\frac{x}{2 c} u\right\} \mathrm{d} u,
\end{aligned}
$$

from which (3.2.4) follows.

3.3. Proof of Theorem 1.1 in the i.i.d. case. In this Subsection, we will prove Theorem 1.1 in the particular case of an i.i.d. random field.

Theorem 3.15. Let $\left(X_{\boldsymbol{i}}\right)_{\boldsymbol{i} \in \mathbb{Z}^{d}}$ be a centered i.i.d. random field. For all $1<p<2$, the following inequality holds:

$$
\left\|\sup _{\boldsymbol{n} \in \mathbb{N}^{d}} \frac{1}{\sqrt{|\boldsymbol{n}| L L(|\boldsymbol{n}|)}}\left|\sum_{\mathbf{1} \preccurlyeq \boldsymbol{i} \preccurlyeq \boldsymbol{n}} X_{\boldsymbol{i}}\right|\right\|_{p} \leqslant c_{p, d}\left\|X_{\mathbf{0}}\right\|_{2, d-1},
$$

where $c_{p, d}$ depends only on $p$ and $d$.

Proof. We follows the ideas of the proof of Theorem 2.3 in [Cun15]. Let us fix $1<p<2$. In view of Proposition 3.13 and Lemma 3.5, it suffices to establish that if $\left\|X_{\mathbf{0}}\right\|_{2, d-1}=1$, then

$$
x^{p} \mathbb{P}\left\{\sup _{\boldsymbol{n} \in \mathbb{N}_{0}} \frac{1}{\sqrt{|\boldsymbol{n}| L L(|\boldsymbol{n}|)}}\left|\sum_{\mathbf{1} \preccurlyeq \boldsymbol{i} \preccurlyeq \boldsymbol{n}} X_{\boldsymbol{i}}\right|>x\right\} \leqslant C_{p, d},
$$

It suffices to prove (3.3.2) for $x$ such that

$$
\frac{x^{2}}{2 x^{p}+2}-d \geqslant \frac{p d}{2-p} \text {. }
$$

Let us fix such an $x$. We will use the notation $s(\boldsymbol{n})=\sum_{q=1}^{d} n_{q}$ for $\boldsymbol{n} \in \mathbb{N}^{d}$. Define the events

$$
A_{\boldsymbol{n}}:=\left\{\frac{1}{\sqrt{2^{s(\boldsymbol{n})} L(s(\boldsymbol{n}))}}\left|\sum_{1 \preccurlyeq i \preccurlyeq 2^{n}} X_{\boldsymbol{i}}\right|>x\right\} ;
$$




$$
B_{\boldsymbol{n}}:=\left\{\frac{1}{2^{s(\boldsymbol{n})}}\left|\sum_{1 \preccurlyeq i \preccurlyeq 2^{n}} X_{\boldsymbol{i}}^{2}\right|>x^{p}\right\} .
$$

It suffices to establish that

$$
\sum_{\boldsymbol{n} \succcurlyeq \mathbf{0}} \mathbb{P}\left(A_{\boldsymbol{n}} \cap B_{\boldsymbol{n}}^{c}\right)+\mathbb{P}\left(\bigcup_{\boldsymbol{n} \succcurlyeq \mathbf{0}} B_{\boldsymbol{n}}\right) \leqslant C_{p, d} x^{-p} .
$$

Let us fix an integer $N$. Define the sets $J:=\{\boldsymbol{n} \succcurlyeq \mathbf{0} \mid s(\boldsymbol{n}) \leqslant N\}$ and $J^{\prime}:=\{\boldsymbol{n} \succcurlyeq \mathbf{0} \mid s(\boldsymbol{n})>N\}$, where $s(\boldsymbol{n})=\sum_{q=1}^{d} n_{q}$. Using Chebyshev's inequality, we get

$$
\sum_{\boldsymbol{n} \in J} \mathbb{P}\left(A_{\boldsymbol{n}} \cap B_{\boldsymbol{n}}^{c}\right) \leqslant x^{-2} \operatorname{Card}(J) \leqslant x^{-2} c_{d} \sum_{k=1}^{N} k^{d-1} \leqslant c_{d} x^{-2} N^{d} .
$$

Now, we control for a fixed $\boldsymbol{n} \in J^{\prime}$ the quantity $\mathbb{P}\left(A_{\boldsymbol{n}} \cap B_{\boldsymbol{n}}^{c}\right)$. Let $\left(d_{i}\right)_{i \in \mathbb{Z}}$ be an i.i.d. sequence such that $d_{1}$ has the same distribution as $X_{1}$. Then

$$
\mathbb{P}\left(A_{\boldsymbol{n}} \cap B_{\boldsymbol{n}}^{c}\right)=\mathbb{P}\left(\left\{\frac{1}{\sqrt{2^{s(\boldsymbol{n})} L(s(\boldsymbol{n}))}}\left|\sum_{j=1}^{2^{s(\boldsymbol{n})}} d_{j}\right|>x\right\} \cap\left\{\frac{1}{2^{s(\boldsymbol{n})}} \sum_{j=1}^{2^{s(\boldsymbol{n})}} d_{j}^{2} \leqslant x^{p}\right\}\right)
$$

and by using Proposition 3.3 in the following context: $n:=2^{s(\boldsymbol{n})}$ and $y=x^{p} 2^{s(\boldsymbol{n})}$, we obtain the bound

$$
\mathbb{P}\left(A_{\boldsymbol{n}} \cap B_{\boldsymbol{n}}^{c}\right) \leqslant 2 \exp \left(-2^{s(\boldsymbol{n})} L(s(\boldsymbol{n})) \frac{x^{2}}{2\left(x^{p 2^{s(\boldsymbol{n})}}+2^{s(\boldsymbol{n})} \mathbb{E}\left[X_{1}^{2}\right]\right)}\right) .
$$

Due to the assumption (3.3.3) and the fact that $\mathbb{E}\left[X_{1}^{2}\right] \leqslant 1$, we obtain the estimate

$$
\mathbb{P}\left(A_{\boldsymbol{n}} \cap B_{\boldsymbol{n}}^{c}\right) \leqslant 2 \exp \left(-L(s(\boldsymbol{n}))\left(\frac{p d}{2-p}+d\right)\right) .
$$

As the number of elements $\boldsymbol{n}$ of $\mathbb{N}^{d}$ such that $s(\boldsymbol{n})=k$ is of order $k^{d-1}$ and $x \geqslant a_{p, d}$, we get that

$$
\mathbb{P}\left(A_{\boldsymbol{n}} \cap B_{\boldsymbol{n}}^{c}\right) \leqslant c_{d} \sum_{k \geqslant N+1} k^{d-1-\left(\frac{p d}{2-p}+d\right)} \leqslant c_{d} \sum_{k \geqslant N+1} k^{-1-\frac{p d}{2-p}} \leqslant c_{p, d} N^{-\frac{p d}{2-p}} .
$$

Using Proposition 3.7, we derive that

$$
\mathbb{P}\left(\bigcup_{n \succcurlyeq 0} B_{n}\right) \leqslant c_{d} x^{-p} \mathbb{E}\left[\varphi_{2, d-1}\left(\left|X_{\mathbf{0}}\right|\right)\right] \leqslant c_{d} x^{-p} .
$$

Choosing $N:=\left\lfloor x^{\frac{2-p}{d}}\right\rfloor$, the previous estimations give (3.3.6). This ends the proof of Theorem 3.15.

3.4. Proof of Theorem 1.1. For $\boldsymbol{n} \in \mathbb{N}^{d}$, define $c_{\boldsymbol{n}}:=\sqrt{|\boldsymbol{n}| L L(|\boldsymbol{n}|)}$. We associate to a random field $\left(X_{i}\right)_{i \in \mathbb{Z}^{d}}$ a maximal function, namely

$$
M\left(\left(X_{i}\right)_{i \in \mathbb{Z}^{d}}\right):=\sup _{\boldsymbol{n} \in \mathbb{N}^{d}} \frac{1}{c_{\boldsymbol{n}}}\left|\sum_{\mathbf{1} \preccurlyeq \boldsymbol{i} \preccurlyeq \boldsymbol{n}} X_{\boldsymbol{i}}\right| .
$$


By the martingale convergence theorem, the decomposition $X_{i}=X_{i, 0}+\sum_{j=1}^{+\infty} X_{i, j}$ holds almost surely where $X_{\boldsymbol{i}, j}=\mathbb{E}\left[X_{\boldsymbol{i}} \mid \sigma\left\{\varepsilon_{\boldsymbol{u}},\|\boldsymbol{u}-\boldsymbol{i}\|_{\infty} \leqslant j\right\}\right]-\mathbb{E}\left[X_{\boldsymbol{i}} \mid \sigma\left\{\varepsilon_{\boldsymbol{u}},\|\boldsymbol{u}-\boldsymbol{i}\|_{\infty} \leqslant j-1\right\}\right]$ hence

$$
M\left(\left(X_{i}\right)_{i \in \mathbb{Z}^{d}}\right) \leqslant M\left(\left(X_{i, 0}\right)_{i \in \mathbb{Z}^{d}}\right)+\sum_{j=1}^{+\infty} M\left(\left(X_{i, j}\right)_{i \in \mathbb{Z}^{d}}\right) .
$$

Therefore, it suffices to control the maximal function associated to each random field $\left(X_{\boldsymbol{i}, j}\right)_{\boldsymbol{i} \in \mathbb{Z}^{d}}$, which has the feature that for all fixed $j, X_{i, j}$ is a function of $\left(\varepsilon_{\boldsymbol{i}-\boldsymbol{u}}\right)_{\|\boldsymbol{u}\|_{\infty} \leqslant j}$. For such a random field, the maximal function can be bounded by a finite sum of maximal functions associated to an i.i.d. random field.

Let $\kappa$ be a constant such that for all $N \succcurlyeq \mathbf{1}, c_{N+1} \leqslant \kappa c_{N}$.

Lemma 3.16. For all positive integer $k_{0}$, the following inequality holds:

$$
M\left(\left(X_{\boldsymbol{i}}\right)_{\boldsymbol{i} \in \mathbb{Z}^{d}}\right) \leqslant \kappa k_{0}^{-d / 2} \sum_{\mathbf{1} \preccurlyeq \boldsymbol{b} \preccurlyeq k_{0} \mathbf{1}} \widetilde{M}\left(\left(X_{k_{0} \boldsymbol{a}+\boldsymbol{b}}\right)_{\boldsymbol{a} \in \mathbb{Z}^{d}}\right),
$$

where

$$
\widetilde{M}\left(\left(X_{i}\right)_{i \in \mathbb{Z}^{d}}\right):=\sup _{\boldsymbol{n} \in \mathbb{N}^{d}} \frac{1}{c_{\boldsymbol{n}}}\left|\sum_{\mathbf{0} \preccurlyeq \boldsymbol{i} \preccurlyeq \boldsymbol{n}} X_{\boldsymbol{i}}\right| .
$$

Observe that the only difference between $M$ and $\widetilde{M}$ lies in the index of summation. If $\left(X_{i}\right)_{i \in \mathbb{Z}^{d}}$ is strictly stationary, then

$$
\left\|\widetilde{M}\left(\left(X_{\boldsymbol{i}}\right)_{\boldsymbol{i} \in \mathbb{Z}^{d}}\right)\right\|_{p}=\left\|\sup _{\boldsymbol{n} \in \mathbb{N}^{d}} \frac{1}{c_{\boldsymbol{n}}}\left|\sum_{\boldsymbol{1} \preccurlyeq \boldsymbol{i} \preccurlyeq \boldsymbol{n}+\mathbf{1}} X_{\boldsymbol{i}}\right|\right\|_{p} \leqslant \kappa\left\|M\left(\left(X_{\boldsymbol{i}}\right)_{\boldsymbol{i} \in \mathbb{Z}^{d}}\right)\right\|_{p} .
$$

Proof. Let

$$
M^{\prime}\left(\left(X_{i}\right)_{i \in \mathbb{Z}^{d}}\right):=\sup _{\boldsymbol{N} \succcurlyeq \mathbf{1}} \frac{1}{c_{k_{0}} \boldsymbol{N}}\left|\sum_{\mathbf{1} \preccurlyeq \boldsymbol{i} \preccurlyeq k_{0} \boldsymbol{N}} X_{\boldsymbol{i}}\right|
$$

If $M$ was replaced by $M^{\prime}$ in the left hand side of (3.4.3), then Lemma 3.16 would follow from a decomposition of $\sum_{\mathbf{1} \preccurlyeq \boldsymbol{i} \preccurlyeq \boldsymbol{n}} X_{\boldsymbol{i}}$ according to the remainder of the Euclidean division by $k_{0}$ of each $i_{q}$. We will link $M$ and $M^{\prime}$. We start from the estimate

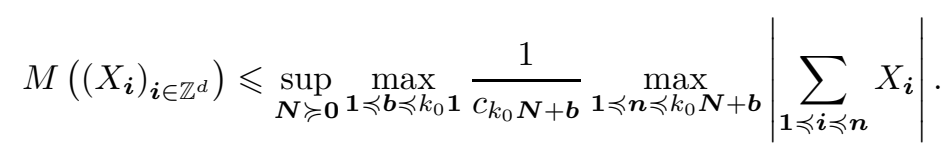

Using the fact that $c_{k_{0} \boldsymbol{N}+\boldsymbol{b}} \geqslant c_{k_{0} \boldsymbol{N}} \geqslant k_{0}^{d / 2} c_{\boldsymbol{N}}$ and the inequality

$$
\max _{\mathbf{1} \preccurlyeq \boldsymbol{b} \preccurlyeq k_{0} \mathbf{1}} \max _{\mathbf{1} \preccurlyeq \boldsymbol{n} \preccurlyeq k_{0} \boldsymbol{N}+\boldsymbol{b}}\left|\sum_{\mathbf{1} \preccurlyeq \boldsymbol{i} \preccurlyeq \boldsymbol{n}} X_{\boldsymbol{i}}\right| \leqslant \max _{\mathbf{1} \preccurlyeq \boldsymbol{n} \preccurlyeq k_{0}(\boldsymbol{N}+\mathbf{1})}\left|\sum_{\mathbf{1} \preccurlyeq \boldsymbol{i} \preccurlyeq \boldsymbol{n}} X_{\boldsymbol{i}}\right|,
$$

we infer that

$$
M\left(\left(X_{\boldsymbol{i}}\right)_{\boldsymbol{i} \in \mathbb{Z}^{d}}\right) \leqslant k_{0}^{-d / 2} \sup _{\boldsymbol{N} \succcurlyeq \mathbf{0}} \frac{1}{c_{N}} \max _{1 \preccurlyeq n \preccurlyeq k_{0}(\boldsymbol{N}+\mathbf{1})}\left|\sum_{\mathbf{1} \preccurlyeq \boldsymbol{i} \preccurlyeq \boldsymbol{n}} X_{\boldsymbol{i}}\right| .
$$


From the definition of $\kappa$, we get

$$
M\left(\left(X_{\boldsymbol{i}}\right)_{\boldsymbol{i} \in \mathbb{Z}^{d}}\right) \leqslant \kappa k_{0}^{-d / 2} \sup _{\boldsymbol{N} \succcurlyeq \mathbf{1}} \frac{1}{c_{\boldsymbol{N}}} \max _{\mathbf{1} \preccurlyeq \boldsymbol{n} \preccurlyeq k_{0}}\left|\sum_{\mathbf{1} \preccurlyeq \boldsymbol{i} \preccurlyeq \boldsymbol{n}} X_{\boldsymbol{i}}\right| .
$$

Using the fact that for all $\boldsymbol{n}$ such that $\mathbf{1} \preccurlyeq \boldsymbol{n} \preccurlyeq k_{0} \boldsymbol{N}$,

$$
\left|\sum_{\mathbf{1} \preccurlyeq \boldsymbol{i} \preccurlyeq \boldsymbol{n}} X_{\boldsymbol{i}}\right| \leqslant \max _{\mathbf{1} \preccurlyeq \boldsymbol{n}^{\prime} \preccurlyeq \boldsymbol{N}} \sum_{\mathbf{1} \preccurlyeq \boldsymbol{b} \preccurlyeq k_{0} \mathbf{1}}\left|\sum_{\mathbf{0} \preccurlyeq \boldsymbol{a} \preccurlyeq \boldsymbol{n}^{\prime}} X_{k_{0} \boldsymbol{a}+\boldsymbol{b}}\right|,
$$

we conclude the proof of Lemma 3.16.

For all fixed $j \geqslant 1$, we apply Lemma 3.16 to $\widetilde{X_{i}}:=X_{i, j}$ and $k_{0}:=4 j+1$ in order to obtain, after having taken the $\mathbb{L}^{p}$-norms,

$$
\begin{aligned}
\left\|M\left(\left(X_{\boldsymbol{i}}\right)_{\boldsymbol{i} \in \mathbb{Z}^{d}}\right)\right\|_{p} \leqslant & \left\|M\left(\left(X_{\boldsymbol{i}, 0}\right)_{\boldsymbol{i} \in \mathbb{Z}^{d}}\right)\right\|_{p} \\
& +\kappa \sum_{j=1}^{+\infty}(4 j+1)^{-d / 2} \sum_{\mathbf{1} \preccurlyeq \boldsymbol{b} \preccurlyeq(4 j+1) \mathbf{1}}\left\|\widetilde{M}\left(\left(X_{(4 j+1) \boldsymbol{a}+\boldsymbol{b}}\right)_{\boldsymbol{a} \in \mathbb{Z}^{d}}\right)\right\|_{p} .
\end{aligned}
$$

We then conclude by applying first (3.4.5) then Theorem 3.15 for all $j \geqslant 1$ and $\boldsymbol{b} \in \mathbb{Z}^{d}$ such that $\mathbf{1} \preccurlyeq \boldsymbol{b} \preccurlyeq(4 j+1) \mathbf{1}$ to the i.i.d. random field $\left(X_{(4 j+1) \boldsymbol{a}+\boldsymbol{b}}\right)_{\boldsymbol{a} \in \mathbb{Z}^{d}}$, and to the i.i.d. random field $\left(X_{\boldsymbol{i}, 0}\right)_{\boldsymbol{i} \in \mathbb{Z}^{d}}$.

This ends the proof of Theorem 1.1.

3.5. Proof of Theorem 1.2. First observe that by definition of linear processes,

$$
\sup _{n \geqslant 1} \frac{1}{\sqrt{\ell_{n} L L\left(\ell_{n}\right)}}\left|\sum_{\boldsymbol{i} \in \Lambda_{n}} X_{\boldsymbol{i}}\right| \leqslant \sum_{\boldsymbol{j} \in \mathbb{Z}^{d}} \sup _{n \geqslant 1} \frac{1}{\sqrt{\ell_{n} L L\left(\ell_{n}\right)}}\left|\sum_{\boldsymbol{i} \in \Lambda_{n}} a_{\boldsymbol{j}} \varepsilon_{\boldsymbol{i}-\boldsymbol{j}}\right|
$$

hence

$$
\left\|\sup _{n \geqslant 1} \frac{1}{\sqrt{\ell_{n} L L\left(\ell_{n}\right)}}\left|\sum_{\boldsymbol{i} \in \Lambda_{n}} X_{\boldsymbol{i}}\right|\right\|_{p} \leqslant \sum_{\boldsymbol{j} \in \mathbb{Z}^{d}}\left|a_{\boldsymbol{j}}\right|\left\|\sup _{n \geqslant 1} \frac{1}{\sqrt{\ell_{n} L L\left(\ell_{n}\right)}} \mid \sum_{\boldsymbol{i} \in \Lambda_{n}-\boldsymbol{j}} \varepsilon_{\boldsymbol{i}}\right\| \|_{p},
$$

where $\Lambda_{n}-\boldsymbol{j}$ is the set of the elements of $\mathbb{Z}^{d}$ of the form $\boldsymbol{i}-\boldsymbol{j}$, where $\boldsymbol{i} \in \Lambda_{n}$. Since $\Lambda_{n}-\boldsymbol{j}$ has the same number of elements as $\Lambda_{n}$, it suffices to prove Theorem 1.2 in the i.i.d. case.

We thus assume that the random field $\left(X_{\boldsymbol{i}}\right)_{\boldsymbol{i} \in \mathbb{Z}^{d}}$ is i.i.d., centered and $\mathbb{E}\left[X_{\mathbf{1}}^{2}\right]=1$. We will essentially follow the steps of the proof Theorem 2.3 in [Cun15]; nervertheless, Theorem 1.2 is not a direct consequence of this result as the sets $\Lambda_{n}$ may not satisfy any inclusion relation. As in the proof of Theorem 1.1, it suffices to prove that for each $1<p<2$, there exists some positive constants $t_{0}$ and $c_{p}$ such that

$$
\sup _{t \geqslant t_{0}} t^{p} \mathbb{P}\left\{\sup _{n \geqslant 1} \frac{1}{\sqrt{\ell_{n} L L\left(\ell_{n}\right)}}\left|\sum_{\boldsymbol{i} \in \Lambda_{n}} X_{\boldsymbol{i}}\right|>t\right\} \leqslant c_{p} C \delta^{-p / 2} .
$$

We will take

$$
t_{0}:=\sqrt{\frac{2}{(2-p) h(1) \delta}}
$$


where $h$ is like in Proposition 3.4. This will give that $\left\|\sup _{n \geqslant 1} \frac{1}{\sqrt{\ell_{n} L L\left(\ell_{n}\right)}}\left|\sum_{\boldsymbol{i} \in \Lambda_{n}} X_{\boldsymbol{i}}\right|\right\|_{p, w} \leqslant$ $\left(c_{p} C \delta^{-p / 2}\right)^{1 / p}+t_{0}$ and using (3.1.2) with $p+\varepsilon$ will give (1.2.5).

In order to show (3.5.3), we use truncation and introduce the random variables

$$
\begin{aligned}
& X_{i, n}:=X_{\boldsymbol{i}} \mathbf{1}\left\{\left|X_{\boldsymbol{i}}\right| \leqslant a t \sqrt{\frac{\ell_{n}}{L L\left(\ell_{n}\right)}}\right\}-\mathbb{E}\left[X_{\boldsymbol{i}} \mathbf{1}\left\{\left|X_{i}\right| \leqslant a t \sqrt{\frac{\ell_{n}}{L L\left(\ell_{n}\right)}}\right\}\right], \\
& X_{i, n}^{\prime}:=X_{\boldsymbol{i}} \mathbf{1}\left\{\left|X_{\boldsymbol{i}}\right|>a t \sqrt{\frac{\ell_{n}}{L L\left(\ell_{n}\right)}}\right\}-\mathbb{E}\left[X_{\boldsymbol{i}} \mathbf{1}\left\{\left|X_{\boldsymbol{i}}\right|>a t \sqrt{\frac{\ell_{n}}{L L\left(\ell_{n}\right)}}\right\}\right],
\end{aligned}
$$

where $t \geqslant t_{0}$ is fixed and $a=(2-p) h(1) \delta / 2$, where $h$ is like in Proposition 3.4. Let $N:=$ $\left\lfloor t^{2-p}\right\rfloor+1$. Then

$$
\begin{aligned}
& \mathbb{P}\left\{\sup _{n \geqslant 1} \frac{1}{\sqrt{\ell_{n} L L\left(\ell_{n}\right)}}\left|\sum_{\boldsymbol{i} \in \Lambda_{n}} X_{\boldsymbol{i}}\right|>2 t\right\} \leqslant \sum_{n=1}^{N} \mathbb{P}\left\{\frac{1}{\sqrt{\ell_{n} L L\left(\ell_{n}\right)}}\left|\sum_{\boldsymbol{i} \in \Lambda_{n}} X_{\boldsymbol{i}}\right|>2 t\right\} \\
& \quad+\sum_{n \geqslant N+1} \mathbb{P}\left\{\frac{1}{\sqrt{\ell_{n} L L\left(\ell_{n}\right)}}\left|\sum_{\boldsymbol{i} \in \Lambda_{n}} X_{\boldsymbol{i}, n}\right|>t\right\}+\sum_{n \geqslant N+1} \mathbb{P}\left\{\frac{1}{\sqrt{\ell_{n} L L\left(\ell_{n}\right)}}\left|\sum_{\boldsymbol{i} \in \Lambda_{n}} X_{\boldsymbol{i}, n}^{\prime}\right|>t\right\} .
\end{aligned}
$$

The term $\sum_{n=1}^{N} \mathbb{P}\left\{\frac{1}{\sqrt{\ell_{n} L L\left(\ell_{n}\right)}}\left|\sum_{i \in \Lambda_{n}} X_{\boldsymbol{i}}\right|>2 t\right\}$ can be controlled by Chebyshev's inequality, independence and the assumption of unit variance of $X_{i}$. We get that

$$
\sum_{n=1}^{N} \mathbb{P}\left\{\frac{1}{\sqrt{\ell_{n} L L\left(\ell_{n}\right)}}\left|\sum_{i \in \Lambda_{n}} X_{i}\right|>2 t\right\} \leqslant \frac{1}{4 t^{2}} N \leqslant \frac{1}{4} t^{-p}+\frac{1}{4 t^{-p} t_{0}^{2-p}} .
$$

In order to control $\mathbb{P}\left\{\frac{1}{\sqrt{\ell_{n} L L\left(\ell_{n}\right)}}\left|\sum_{\boldsymbol{i} \in \Lambda_{n}} X_{\boldsymbol{i}, n}\right|>t\right\}$ for $n \geqslant N+1$, we apply Proposition 3.4 in the following setting: $c=\frac{2 a t \sqrt{\ell_{n}}}{\sqrt{L L\left(\ell_{n}\right)}}, x=t \sqrt{\ell_{n} L L\left(\ell_{n}\right)}$ and $y=t^{2} a \ell_{n}$. We obtain that

$$
\sum_{n \geqslant N+1} \mathbb{P}\left\{\frac{1}{\sqrt{\ell_{n} L L\left(\ell_{n}\right)}}\left|\sum_{i \in \Lambda_{n}} X_{i, n}\right|>t\right\} \leqslant 2 \sum_{n \geqslant N+1} \exp \left(-\frac{h(1)}{a} L L\left(\ell_{n}\right)\right) .
$$

From the assumption $\ell_{n} \geqslant \exp \left(n^{\delta}\right)$, we get that

$$
\sum_{n \geqslant N+1} \mathbb{P}\left\{\frac{1}{\sqrt{\ell_{n} L L\left(\ell_{n}\right)}}\left|\sum_{i \in \Lambda_{n}} X_{i, n}\right|>t\right\} \leqslant 2 \sum_{n \geqslant N+1} \exp \left(-\frac{h(1) \delta}{a} L(n)\right) .
$$

One gets that the contribution of the previous term does not exceed $4 t^{-p} /(2-p)$. It remains to control $\sum_{n \geqslant N+1} \mathbb{P}\left\{\frac{1}{\sqrt{\ell_{n} L L\left(\ell_{n}\right)}}\left|\sum_{i \in \Lambda_{n}} X_{i, n}^{\prime}\right|>t\right\}$. To do so, we start from Markov's inequality:

$$
\sum_{n \geqslant N+1} \mathbb{P}\left\{\frac{1}{\sqrt{\ell_{n} L L\left(\ell_{n}\right)}}\left|\sum_{\boldsymbol{i} \in \Lambda_{n}} X_{i, n}^{\prime}\right|>t\right\} \leqslant \sum_{n \geqslant N+1} \frac{1}{t \sqrt{\ell_{n} L L\left(\ell_{n}\right)}} \sum_{\boldsymbol{i} \in \Lambda_{n}} \mathbb{E}\left[\left|X_{\boldsymbol{i}, n}^{\prime}\right|\right]
$$


and by definition of $X_{\boldsymbol{i}, n}^{\prime}$, we get that

$$
\sum_{n \geqslant N+1} \mathbb{P}\left\{\frac{1}{\sqrt{\ell_{n} L L\left(\ell_{n}\right)}}\left|\sum_{i \in \Lambda_{n}} X_{i, n}^{\prime}\right|>t\right\} \leqslant \frac{2}{t} \sum_{n \geqslant 1} b_{n} \mathbb{E}\left[\left|X_{\mathbf{1}}\right| \mathbf{1}\left\{\left|X_{\mathbf{1}}\right|>a t b_{n}\right\}\right],
$$

where $b_{n}:=\frac{2^{n / 2}}{\sqrt{L(n)}}$. For a random variable $X$,

$$
\sum_{n \geqslant 1} b_{n} 1\left\{|X|>b_{n}\right\}=\sum_{n \geqslant 1} b_{n} \sum_{k \geqslant n} 1\left\{b_{k}<|X| \leqslant b_{k+1}\right\} \leqslant C|X|,
$$

as $\sum_{n=1}^{k} b_{n} \leqslant C b_{k}$. Applying this to $X=\left|X_{1}\right| /(a t)$ gives that

$$
\sum_{n \geqslant N+1} \mathbb{P}\left\{\frac{1}{\sqrt{\ell_{n} L L\left(\ell_{n}\right)}}\left|\sum_{i \in \Lambda_{n}} X_{i, n}^{\prime}\right|>t\right\} \leqslant \frac{4 C}{(2-p) \delta h(1) t^{p} t_{0}^{2-p}}
$$

and we get (3.5.3) in view of the previous estimates. This ends the proof of Theorem 1.2.

3.6. Proof of Theorem 1.3. From the result of the previous Subsection, we got that for an i.i.d. centered random field $\left(Y_{\boldsymbol{i}}\right)_{\boldsymbol{i} \in \mathbb{Z}^{d}}$ and subsets $\Lambda_{n}$ of $\mathbb{Z}^{d}$ of cardinal $\ell_{n}$

$$
\left\|\sup _{n \geqslant 1} \frac{1}{\sqrt{\ell_{n} L L\left(\ell_{n}\right)}} \mid \sum_{i \in \Lambda_{n}} Y_{\boldsymbol{i}}\right\|\left\|_{p} \leqslant C_{p} C^{1 / p} \delta^{-1 / 2}\right\| Y_{\mathbf{0}} \|_{2},
$$

provided that $\ell_{n+1} \geqslant \ell_{n} \geqslant \exp \left(n^{\delta}\right)$ and $\sum_{k=1}^{n} \sqrt{\ell_{k} / L L\left(\ell_{k}\right)} \leqslant C \ell_{n} / L L\left(\ell_{n}\right)$. By the martingale convergence theorem, the decomposition $X_{i}=X_{i, 0}+\sum_{j=1}^{+\infty} X_{i, j}$ holds almost surely where $X_{\boldsymbol{i}, j}=\mathbb{E}\left[X_{\boldsymbol{i}} \mid \sigma\left\{\varepsilon_{\boldsymbol{u}},\|\boldsymbol{u}-\boldsymbol{i}\|_{\infty} \leqslant j\right\}\right]-\mathbb{E}\left[X_{\boldsymbol{i}} \mid \sigma\left\{\varepsilon_{\boldsymbol{u}},\|\boldsymbol{u}-\boldsymbol{i}\|_{\infty} \leqslant j-1\right\}\right]$ hence

$$
\left\|\operatorname { s u p } _ { n \geqslant 1 } \frac { 1 } { \sqrt { \ell _ { n } L L ( \ell _ { n } ) } } \left|\sum_{i \in \Lambda_{n}} X_{\boldsymbol{i}}\|\|_{p} \leqslant \sum_{j \geqslant 0}\left\|\sup _{n \geqslant 1} \frac{1}{\sqrt{\ell_{n} L L\left(\ell_{n}\right)}} \mid \sum_{\boldsymbol{i} \in \Lambda_{n}} X_{\boldsymbol{i}, j}\right\| \|_{p} .\right.\right.
$$

For each fixed $j \geqslant 1, X_{\boldsymbol{i}, j}$ is a function of the random variables $\varepsilon_{\boldsymbol{u}}$, where $\boldsymbol{u}$ runs over the $\boldsymbol{u} \in \mathbb{Z}^{d}$ such that $\|\boldsymbol{u}-\boldsymbol{i}\| \leqslant j$. Although $\left(X_{\boldsymbol{i}, j}\right)_{\boldsymbol{i} \in \mathbb{Z}^{d}}$ is not an independent random field, one can cut the summation over $\Gamma_{n}$ according to the remainder with respect to the Euclidean division of each coordinate by $4 j+2$. Indeed, for $j \geqslant 1$ fixed, define the sets

$$
\Gamma_{n}^{\boldsymbol{a}, j}:=\left\{\boldsymbol{i} \in \mathbb{Z}^{d},(4 j+2) \mathbf{1}+\boldsymbol{a} \in \Gamma_{n}\right\}, \boldsymbol{a}=\left(a_{q}\right)_{q=1}^{d}, 0 \leqslant a_{q} \leqslant 4 j+1
$$

and the random fields $\left(Y_{\boldsymbol{i}, j}^{\boldsymbol{a}}\right)_{\boldsymbol{i} \in \mathbb{Z}}$ by

$$
Y_{\boldsymbol{i}, j}^{\boldsymbol{a}}=X_{(4 j+2) \boldsymbol{i}+\boldsymbol{a}, j} .
$$

Then the following inequality holds:

$$
\left\|\operatorname { s u p } _ { n \geqslant 1 } \frac { 1 } { \sqrt { \ell _ { n } L L ( \ell _ { n } ) } } \left|\sum_{\boldsymbol{i} \in \Gamma_{n}} X_{\boldsymbol{i}, j}\|\|_{p} \leqslant \sum_{\mathbf{0} \preccurlyeq \boldsymbol{a} \preccurlyeq(4 j+1) \mathbf{1}}\left\|\sup _{n \geqslant 1} \frac{1}{\sqrt{\ell_{n} L L\left(\ell_{n}\right)}}\left|\sum_{\boldsymbol{i} \in \Gamma_{n}^{\boldsymbol{a}}} Y_{\boldsymbol{i}, j}^{\boldsymbol{a}}\right|\right\|_{p} .\right.\right.
$$


Now, we have to show that the sets $\Gamma_{n}^{\boldsymbol{a}, j}$ also satisfy the assumptions $\ell_{n+1}^{\boldsymbol{a}, j} \geqslant \ell_{n}^{\boldsymbol{a}, j} \geqslant \exp \left(n_{\boldsymbol{a}, j}^{\delta}\right)$ and $\sum_{k=1}^{n} \sqrt{\ell_{k}^{\boldsymbol{a}, j} / L L\left(\ell_{k}^{\boldsymbol{a}, j}\right)} \leqslant C_{\boldsymbol{a}, j} \ell_{n}^{\boldsymbol{a}, j} / L L\left(\ell_{n}^{\boldsymbol{a}, j}\right)$, where $\ell_{n}^{\boldsymbol{a}, j}$ denotes the cardinal of $\Gamma_{n}^{\boldsymbol{a}, j}$. Define

$$
\Gamma_{n}^{\boldsymbol{a}, j}(w):=\left\{\boldsymbol{i} \in \mathbb{Z}^{d},(4 j+2) \mathbf{1}+\boldsymbol{a} \in \Gamma_{n}(w)\right\}
$$

By assumption,

$$
\ell_{n}^{\boldsymbol{a}, j}=\sum_{w=1}^{J_{n}} \operatorname{Card}\left(\Gamma_{n}^{\boldsymbol{a}}(w)\right)
$$

Moreover,

$$
\operatorname{Card}\left(\Gamma_{n}^{\boldsymbol{a}, j}(w)\right)=\prod_{q=1}^{d}\left(\left\lfloor\frac{\bar{n}_{q}(w, n)-a_{q}}{4 j+2}\right\rfloor-\left\lfloor\frac{\underline{n}_{q}(w, n)-a_{q}}{4 j+2}\right\rfloor+1\right)
$$

hence by disjointness of $\Gamma_{n}^{a}(w), 1 \leqslant w \leqslant J_{n}$ and the assumption $\bar{n}_{q}(w, n)-\underline{n}_{q}(w, n) \geqslant 4$ we derive that

$$
\frac{1}{(4 j+2)^{d} 4^{d}} \ell_{n} \leqslant \ell_{n}^{\boldsymbol{a}, j} \leqslant \frac{1}{(4 j+2)^{d}} \ell_{n} .
$$

In view of (3.6.2) and (3.6.5), we derive that

$$
\begin{aligned}
& \left\|\sup _{n \geqslant 1} \frac{1}{\sqrt{\ell_{n} L L\left(\ell_{n}\right)}}\left|\sum_{\boldsymbol{i} \in \Lambda_{n}} X_{\boldsymbol{i}}\right|\right\|_{p} \\
& \leqslant \sum_{j \geqslant 0} \frac{1}{(4 j+2)^{d / 2} 4^{d / 2}} \sum_{\mathbf{0} \preccurlyeq \boldsymbol{a} \preccurlyeq(4 j+1) \mathbf{1}}\left\|\sup _{n \geqslant 1} \frac{1}{\sqrt{\ell_{n}^{\boldsymbol{a}, j} L L\left(\ell_{n}^{\boldsymbol{a}, j}\right)}}\left|\sum_{\boldsymbol{i} \in \Gamma_{n}^{\boldsymbol{a}, j}} Y_{\boldsymbol{i}, j}^{\boldsymbol{a}}\right|\right\|_{p} .
\end{aligned}
$$

We now apply Theorem 1.2 for each $j \geqslant 0$ and each $\boldsymbol{a} \in \mathbb{Z}^{d}$ such that $\mathbf{0} \preccurlyeq \boldsymbol{a} \preccurlyeq(4 j+1) \mathbf{1}$ in the independent setting, that is, $a_{\mathbf{0}}=1$ and $a_{\boldsymbol{i}}=0$ for $\boldsymbol{i} \neq \mathbf{0}$. In view of (3.6.9), for each $j \geqslant 0$, we may take $\delta_{j}:=(4 j+2)^{-d} 4^{-d}$. For $C_{j}$, we take $C 4^{d}\left(1+\log \left(1+(4 j+1)^{d}\right)+L L\left(e^{2}(4 j+1)^{d}\right)\right)$, $\operatorname{assup}_{s>0} L L(s t) / L L(s) \leqslant 1+(1+\log t)+L L\left(e^{2} t\right)$. This completes the proof of Theorem 1.3.

3.7. Proof of Corollary 2.1. In view of Corollary 1.5, it suffices to estimate $\delta_{d-1}(\boldsymbol{i})$, that is, $\left\|f\left(\left(\varepsilon_{\boldsymbol{i}-\boldsymbol{u}}\right)_{\boldsymbol{u} \in \mathbb{Z}^{d}}\right)-f\left(\left(\varepsilon_{\boldsymbol{i}-\boldsymbol{u}}^{*}\right)_{\boldsymbol{u} \in \mathbb{Z}^{d}}\right)\right\|_{2, d-1}$ where $f=g \circ h$ and $h\left(\left(x_{\boldsymbol{u}}\right)_{\boldsymbol{u} \in \mathbb{Z}^{d}}\right)=\sum_{\boldsymbol{u} \in \mathbb{Z}^{d}} a_{\boldsymbol{u}} x_{\boldsymbol{u}}$ if $\sum_{\boldsymbol{u} \in \mathbb{Z}^{d}} a_{\boldsymbol{u}} x_{\boldsymbol{u}}$ converges (in the sense that $\lim _{m \rightarrow+\infty} \sum_{\substack{\boldsymbol{u} \in \mathbb{Z}^{d} \\\|\|_{\infty} \leqslant m}} a_{\boldsymbol{u}} x_{\boldsymbol{u}}$ exists) and 0 otherwise. Since $g$ is $\gamma$-Hölder continuous, the following inequality holds:

$$
\begin{aligned}
\mathbb{E}\left[\varphi_{2, d-1}\left(f\left(\left(\varepsilon_{\boldsymbol{i}-\boldsymbol{u}}\right)_{\boldsymbol{u} \in \mathbb{Z}^{d}}\right)-f\left(\left(\varepsilon_{\boldsymbol{i}-\boldsymbol{u}}^{*}\right)_{\boldsymbol{u} \in \mathbb{Z}^{d}}\right)\right)\right] & \\
& \leqslant \mathbb{E}\left[\varphi_{2, d-1}\left(\left|h\left(\left(\varepsilon_{\boldsymbol{i}-\boldsymbol{u}}\right)_{\boldsymbol{u} \in \mathbb{Z}^{d}}\right)-h\left(\left(\varepsilon_{\boldsymbol{i}-\boldsymbol{u}}^{*}\right)_{\boldsymbol{u} \in \mathbb{Z}^{d}}\right)\right|^{\gamma}\right)\right]
\end{aligned}
$$

hence

$$
\mathbb{E}\left[\varphi_{2, d-1}\left(\frac{f\left(\left(\varepsilon_{\boldsymbol{i}-\boldsymbol{u}}\right)_{\boldsymbol{u} \in \mathbb{Z}^{d}}\right)-f\left(\left(\varepsilon_{\boldsymbol{i}-\boldsymbol{u}}^{*}\right)_{\boldsymbol{u} \in \mathbb{Z}^{d}}\right)}{\lambda}\right)\right] \leqslant \mathbb{E}\left[\varphi_{2, d-1}\left(\frac{\left|a_{\boldsymbol{i}}\right|^{\gamma}\left|\varepsilon_{\mathbf{0}}-\varepsilon_{\mathbf{0}}^{\prime}\right|^{\gamma}}{\lambda}\right)\right] .
$$


Then, using the inequality $\varphi_{2, d-1}\left(t^{\gamma}\right) \leqslant c_{d, \gamma} \varphi_{2 \gamma, d-1}(t)$, we derive that $\delta_{d-1}(\boldsymbol{i}) \leqslant K_{d, \gamma}\left|a_{\boldsymbol{i}}\right|^{\gamma}\left\|\varepsilon_{\mathbf{0}}\right\|_{2 \gamma, d-1}$, which ends the proof of Corollary 2.1 .

3.8. Proof of Corollary 2.3. Here again, we have to estimate $\delta_{d-1}(\boldsymbol{i})$. We will essentially follows the steps given in Example 3 of [BD14], where a bound on the physical dependence measure with the $\mathbb{L}^{p}$-norm instead of the one given by $\varphi_{2, d-1}$ was obtained.

First, according to Theorem 1.1.1 of [Nua09], the expansions

$$
f\left(Y_{\boldsymbol{j}}\right)=\sum_{q=1}^{+\infty} c_{q}(f) H_{q}\left(Y_{\boldsymbol{j}}\right), \quad f\left(Y_{\boldsymbol{j}}^{*}\right)=\sum_{q=1}^{+\infty} c_{q}(f) H_{q}\left(Y_{\boldsymbol{j}}^{*}\right)
$$

hold in $\mathbb{L}^{2}$ hence after having extracted almost surely convergent subsequences $\left(\sum_{q=1}^{Q_{k}} c_{q}(f) H_{q}\left(Y_{j}\right)\right)_{k \geqslant 1}$ and $\left(\sum_{q=1}^{Q_{k}} c_{q}(f) H_{q}\left(Y_{j}^{*}\right)\right)_{k \geqslant 1}$, we derive by Fatou's lemma that

$$
\begin{array}{r}
\left\|f\left(Y_{\boldsymbol{j}}\right)-f\left(Y_{\boldsymbol{j}}^{*}\right)\right\|_{2, d-1} \leqslant \liminf _{k \rightarrow+\infty}\left\|\sum_{q=1}^{Q_{k}} c_{q}(f)\left(H_{q}\left(Y_{\boldsymbol{j}}\right)-H_{q}\left(Y_{\boldsymbol{j}}^{*}\right)\right)\right\|_{2, d-1} \\
\leqslant \sum_{q=1}^{+\infty}\left|c_{q}(f)\right|\left\|H_{q}\left(Y_{\boldsymbol{j}}\right)-H_{q}\left(Y_{j}^{*}\right)\right\|_{2, d-1} .
\end{array}
$$

Therefore, it suffices to prove that there exists a constant $C$ depending only on $d$ such that

$$
\left\|H_{q}\left(Y_{j}\right)-H_{q}\left(Y_{j}^{*}\right)\right\|_{2, d-1} \leqslant C \sqrt{q !} q^{d-\frac{1}{2}}\left\|Y_{j}-Y_{j}^{*}\right\|_{2} .
$$

First, as noticed in Example 3 of [BD14], the following inequality holds for all $q$ :

$$
\left\|H_{q}\left(Y_{\boldsymbol{j}}\right)-H_{q}\left(Y_{\boldsymbol{j}}^{*}\right)\right\|_{2} \leqslant \sqrt{q} \sqrt{q !}\left\|Y_{\boldsymbol{j}}-Y_{\boldsymbol{j}}^{*}\right\|_{2},
$$

because $Y_{\boldsymbol{j}}$ and $Y_{j}^{*}$ have unit variance. By hypercontractivity properties (see [LT91], p. 65), the following inequality holds for all $p \geqslant 2$ :

$$
\left\|H_{q}\left(Y_{j}\right)-H_{q}\left(Y_{j}^{*}\right)\right\|_{p} \leqslant(p-1)^{q / 2}\left\|H_{q}\left(Y_{j}\right)-H_{q}\left(Y_{j}^{*}\right)\right\|_{2} .
$$

Applying (3.8.5) with $p=2+q^{-1}$, taking into account that $\left(1+q^{-1}\right)^{q / 2} \leqslant e^{1 / 2}$ and using (3.8.4) gives

$$
\left\|H_{q}\left(Y_{j}\right)-H_{q}\left(Y_{j}^{*}\right)\right\|_{2+q^{-1}} \leqslant \sqrt{e q \cdot q !}\left\|Y_{j}-Y_{j}^{*}\right\|_{2} .
$$

Observe that for all $u \geqslant 0,(1+\ln (1+u))^{d-1} \leqslant c_{d}(u+1)$; applying this to $u=v^{1 / q}$ and using the inequality $\ln \left(1+v^{1 / q}\right) \geqslant \ln \left((1+v)^{1 / q}\right)=q^{-1} \ln (1+v)$, we derive that

$$
\varphi_{2, d-1}(v) \leqslant c_{d} q^{d-1}\left(v^{2}+v^{2+1 / q}\right)
$$

hence for each random variable $X$, the inequality $\|X\|_{2, d-1} \leqslant c_{d} q^{d-1}\|X\|_{2+q^{-1}}$ holds. Applying this estimate with $X=H_{q}\left(Y_{\boldsymbol{j}}\right)-H_{q}\left(Y_{\boldsymbol{j}}^{*}\right)$ and combining with (3.8.6) gives (3.8.3), which ends the proof of Corollary 2.3. 
3.9. Proof of Corollary 2.5. Let us fix $j \geqslant 1$ and denote $\mathcal{G}$ the $\sigma$-algebra generated by the random variables $\varepsilon_{\boldsymbol{u}},\|\boldsymbol{u}\|_{\infty} \leqslant j$ and define for $\boldsymbol{s}_{\mathbf{1}}, \boldsymbol{s}_{\mathbf{2}} \in \mathbb{Z}^{d}$ such that $\boldsymbol{s}_{\mathbf{1}} \neq \boldsymbol{s}_{\mathbf{2}}$ the random variable

$$
Y_{\boldsymbol{s}_{1}, s_{2}}:=\mathbb{E}\left[\varepsilon_{-s_{1}} \varepsilon_{-s_{2}} \mid \mathcal{G}\right] .
$$

Case 1: $\left\|s_{\mathbf{1}}\right\|_{\infty} \geqslant j+1$ and $\left\|s_{\mathbf{2}}\right\|_{\infty} \geqslant j+1$. The random variable $\varepsilon_{-\boldsymbol{s}_{1},-\boldsymbol{s}_{\mathbf{2}}}$ is independent of $\mathcal{G}$ hence $Y_{\boldsymbol{s}_{1}, \boldsymbol{s}_{2}}:=\mathbb{E}\left[\varepsilon_{-s_{1}} \varepsilon_{-s_{2}}\right]$. Since $\boldsymbol{s}_{1} \neq \boldsymbol{s}_{\mathbf{2}}$ the random variables $\varepsilon_{-s_{1}}$ and $\varepsilon_{-s_{2}}$ are independent, we derive that $Y_{\boldsymbol{s}_{1}, \boldsymbol{s}_{2}}=0$.

Case 2: $\left\|\boldsymbol{s}_{\mathbf{1}}\right\|_{\infty} \geqslant j+1$ and $\left\|\boldsymbol{s}_{\mathbf{2}}\right\|_{\infty} \leqslant j$. Since $\varepsilon_{-\boldsymbol{s}_{\mathbf{2}}}$ is $\mathcal{G}$-measurable and $\varepsilon_{-\boldsymbol{s}_{\mathbf{1}}}$ is independent of $\mathcal{G}$, it follows that $Y_{\boldsymbol{s}_{1}, s_{2}}=0$.

Case 3: $\left\|\boldsymbol{s}_{\mathbf{2}}\right\|_{\infty} \geqslant j+1$ and $\left\|\boldsymbol{s}_{\mathbf{1}}\right\|_{\infty} \leqslant j$. Similarly as in case $2, Y_{\boldsymbol{s}_{\mathbf{1}}, \boldsymbol{s}_{\mathbf{2}}}=0$.

Case 4: $\left\|s_{1}\right\|_{\infty} \leqslant j$ and $\left\|s_{2}\right\|_{\infty} \leqslant j$. Then $\varepsilon_{-s_{1}}$ and $\varepsilon_{-s_{2}}$ are both $\mathcal{G}$-measurable hence $Y_{s_{1}, s_{2}}=\varepsilon_{-s_{1}} \varepsilon_{-s_{2}}$.

Therefore,

$$
\mathbb{E}\left[X_{\mathbf{0}} \mid \sigma\left(\varepsilon_{\boldsymbol{u}}, \boldsymbol{u} \in \mathbb{Z}^{d},\|\boldsymbol{u}\|_{\infty} \leqslant j\right)\right]=\sum_{\substack{\boldsymbol{s}_{\mathbf{1}}, \boldsymbol{s}_{\mathbf{2}} \in \mathbb{Z}^{d} \\\left\|\boldsymbol{s}_{1}\right\|_{\infty} \leqslant j,\left\|\boldsymbol{s}_{\mathbf{2}}\right\|_{\infty} \leqslant j}} a_{\boldsymbol{s}_{1}, \boldsymbol{s}_{\mathbf{2}}} \varepsilon_{-\boldsymbol{s}_{1}} \varepsilon_{-\boldsymbol{s}_{\mathbf{2}}}
$$

and consequently,

$$
\begin{aligned}
X_{\mathbf{0}, j}= & \sum_{\substack{\boldsymbol{s}_{1} \in \mathbb{Z}^{d} \\
\left\|\boldsymbol{s}_{\mathbf{1}}\right\|_{\infty} \leqslant j-1}} \sum_{\substack{\boldsymbol{s}_{\mathbf{2}} \in \mathbb{Z}^{d} \\
\left\|\boldsymbol{s}_{2}\right\|_{\infty}=j,}} a_{\boldsymbol{s}_{1}, \boldsymbol{s}_{\mathbf{2}}} \varepsilon_{-\boldsymbol{s}_{1}} \varepsilon_{-\boldsymbol{s}_{\mathbf{2}}} \\
& +\sum_{\substack{\boldsymbol{s}_{1} \in \mathbb{Z}^{d} \\
\left\|\boldsymbol{s}_{1}\right\|_{\infty}=j}} \sum_{\substack{\boldsymbol{s}_{2} \in \mathbb{Z}^{d} \\
\left\|\boldsymbol{s}_{2}\right\|_{\infty} \leqslant j-1}} a_{\boldsymbol{s}_{1}, \boldsymbol{s}_{\mathbf{2}}} \varepsilon_{-\boldsymbol{s}_{1}} \varepsilon_{-\boldsymbol{s}_{2}}+\sum_{\substack{\boldsymbol{s}_{1} \in \mathbb{Z}^{d} \\
\left\|\boldsymbol{s}_{1}\right\|_{\infty}=j}} \sum_{\substack{\boldsymbol{s}_{2} \in \mathbb{Z}^{d} \\
\left\|\boldsymbol{s}_{2}\right\|_{\infty}=j}} a_{\boldsymbol{s}_{1}, \boldsymbol{s}_{2}} \varepsilon_{-\boldsymbol{s}_{1}} \varepsilon_{-\boldsymbol{s}_{2}} .
\end{aligned}
$$

Let us control the $\mathbb{L}_{2, d-1}$-norm of the first term of the right hand side. Let $I$ be the set of the elements of $\mathbb{Z}^{d}$ whose $\ell^{\infty}$ norm is equal to $j$ and let $\tau:\{1, \ldots$, Card $(I)\} \rightarrow I$ be a bijection. Define the random variable

$$
d_{k}:=\sum_{\substack{\boldsymbol{s}_{1} \in \mathbb{Z}^{d} \\\left\|\boldsymbol{s}_{1}\right\|_{\infty} \leqslant j-1}} a_{\boldsymbol{s}_{\mathbf{1}}, \boldsymbol{\tau}(\boldsymbol{k})} \varepsilon_{-\boldsymbol{s}_{\mathbf{1}}} \varepsilon_{-\boldsymbol{\tau}(\boldsymbol{k})}
$$

and the $\sigma$-algebras

$$
\begin{gathered}
\mathcal{F}_{0}:=\sigma\left(\varepsilon_{\boldsymbol{u}}, \boldsymbol{u} \in \mathbb{Z}^{d},\|\boldsymbol{u}\|_{\infty} \leqslant j-1\right) ; \\
\mathcal{F}_{k}:=\sigma\left(\varepsilon_{\boldsymbol{u}}, \boldsymbol{u} \in \mathbb{Z}^{d},\|\boldsymbol{u}\|_{\infty} \leqslant j-1\right) \vee \sigma\left(\varepsilon_{-\boldsymbol{\tau}\left(k^{\prime}\right)}, 1 \leqslant k^{\prime} \leqslant k\right) .
\end{gathered}
$$

The sequence $\left(d_{k}\right)_{1 \leqslant k \leqslant \operatorname{Card}(I)}$ is a martingale differences sequence with respect to the filtration $\left(\mathcal{F}_{k}\right)_{0 \leqslant k \leqslant \operatorname{Card}(I)}$. Moreover, using independence between $\sum_{\substack{\boldsymbol{s}_{1} \|_{\infty} \in \mathbb{Z}^{d} \leqslant j-1 \\ \boldsymbol{s}_{1}, \boldsymbol{\tau}(\boldsymbol{k})}} \varepsilon_{-\boldsymbol{s}_{1}}$ and $\varepsilon_{-\tau(k)}$ and the inequality $\varphi_{2, d-1}(u v) \leqslant 2^{d-1} \varphi_{2, d-1}(u) \varphi_{2, d-1}(v)$, we derive that $\left\|d_{k}\right\|_{2, d-1} \leqslant$ $2^{d-1}\left\|\varepsilon_{\mathbf{0}}\right\|_{2, d-1}\left\|\sum_{\substack{\boldsymbol{s}_{1} \in \mathbb{Z}^{d} \\\left\|\boldsymbol{s}_{\mathbf{1}}\right\|_{\infty} \leqslant j-1}} a_{\boldsymbol{s}_{\mathbf{1}}, \boldsymbol{\tau}(\boldsymbol{k})} \varepsilon_{-\boldsymbol{s}_{\mathbf{1}}}\right\|_{2, d-1}$. Since

$$
\mathbb{E}\left[d_{k}^{2} \mid \mathcal{F}_{k-1}\right]=\mathbb{E}\left[\varepsilon_{\mathbf{0}}^{2}\right]\left(\sum_{\substack{\boldsymbol{s}_{1} \in \mathbb{Z}^{d} \\\left\|\boldsymbol{s}_{1}\right\|_{\infty} \leqslant j-1}} a_{\boldsymbol{s}_{\mathbf{1}}, \boldsymbol{\tau}(\boldsymbol{k})} \varepsilon_{-\boldsymbol{s}_{\mathbf{1}}}\right)^{2},
$$


Corollary 3.11 implies that

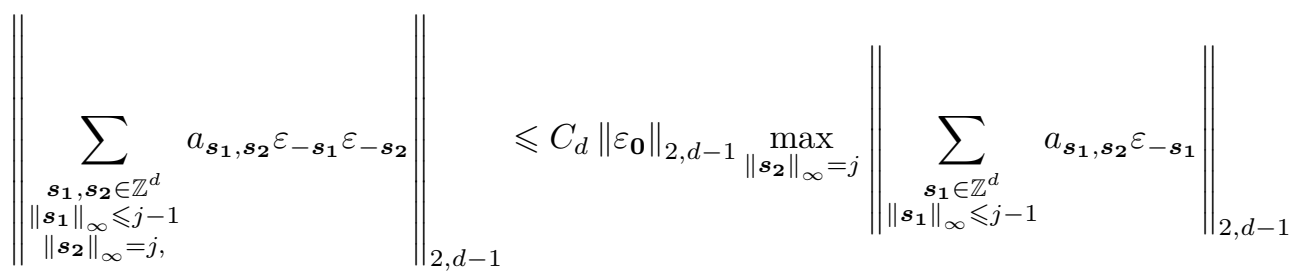

$$
\begin{aligned}
& +C_{d}\left\|\varepsilon_{\mathbf{0}}\right\|_{2}\left(\sum_{\left\|\boldsymbol{s}_{\mathbf{2}}\right\|_{\infty}=j}\left\|\sum_{\substack{\boldsymbol{s}_{\mathbf{1}} \in \mathbb{Z}^{d} \\
\left\|\boldsymbol{s}_{\mathbf{1}}\right\|_{\infty} \leqslant j-1}} a_{\boldsymbol{s}_{\mathbf{1}}, \boldsymbol{\tau}(\boldsymbol{k})} \varepsilon_{-\boldsymbol{s}_{\mathbf{1}}}^{2}\right\|^{1 / 2}\right)^{1, r}
\end{aligned}
$$

which entails, by Lemma 3.9, that

$$
\begin{aligned}
& \left\|\sum_{\substack{\boldsymbol{s}_{1} \in \mathbb{Z}^{d} \\
\left\|\boldsymbol{s}_{1}\right\|_{\infty} \leqslant j-1}} \sum_{\substack{\boldsymbol{s}_{\mathbf{2}} \in \mathbb{Z}^{d} \\
\left\|\boldsymbol{s}_{\mathbf{2}}\right\|_{\infty}=j}} a_{\boldsymbol{s}_{\mathbf{1}}, \boldsymbol{s}_{\mathbf{2}}} \varepsilon_{-\boldsymbol{s}_{\mathbf{1}}} \varepsilon_{-\boldsymbol{s}_{\mathbf{2}}}\right\|_{2, d-1} \\
& \leqslant C_{d}\left\|\varepsilon_{\mathbf{0}}\right\|_{2, d-1}\left(\sum_{\left\|s_{\mathbf{2}}\right\|_{\infty}=j}\left\|\sum_{\substack{\boldsymbol{s}_{\mathbf{1}} \in \mathbb{Z}^{d} \\
\left\|\boldsymbol{s}_{\mathbf{1}}\right\|_{\infty} \leqslant j-1}} a_{\boldsymbol{s}_{\mathbf{1}}, \boldsymbol{s}_{\mathbf{2}}} \varepsilon_{-\boldsymbol{s}_{\mathbf{1}}}\right\|^{2} \|^{1 / 2}\right)^{1 / 2} .
\end{aligned}
$$

We conclude by applying an other time Corollary 3.11.

3.10. Proof of Corollaries 2.2, 2.4 and 2.6. These corollaries follow from Corollary 1.6 and an estimation of the dependence coefficients in the same spirit as in the proof of Corollaries 2.1, 2.3 and 2.6. For the sake of completeness, we give only the key steps of the proof that need to be modified.

For Corollary 2.2, we only need to use a version of (3.7.1) with $\varphi_{2, d-1}$ replaced by $\varphi_{2,0}$.

For Corollary 2.4, we can use directly (3.8.4).

For Corollary 2.6, we can simply use orthogonality of the increments of a martingale difference sequence instead of the inequality given in Corollary 3.11 .

Acknowledgement This research work is supported by the DFG Collaborative Research Center SFB 823 'Statistical modelling of nonlinear dynamic processes'.

The author would like to thank the referee for remarks improving the quality of the manuscript and for the suggestion of considering the summation on disjoin unions of rectangles.

\section{REFERENCES}

[BD79] A. K. Basu and C. C. Y. Dorea, On functional central limit theorem for stationary martingale random fields, Acta Math. Acad. Sci. Hungar. 33 (1979), no. 3-4, 307-316. MR 542479 (80k:60037) 1

[BD14] H. Biermé and O. Durieu, Invariance principles for self-similar set-indexed random fields, Trans. Amer. Math. Soc. 366 (2014), no. 11, 5963-5989. MR 3256190 2, 4, 22 
[BT08] B. Bercu and A. Touati, Exponential inequalities for self-normalized martingales with applications, Ann. Appl. Probab. 18 (2008), no. 5, 1848-1869. MR 24625519

[Bul95] A. V. Bulinskiur, The functional law of the iterated logarithm for associated random fields, Fundam. Prikl. Mat. 1 (1995), no. 3, 623-639. MR 17885462

[Bur73] D. L. Burkholder, Distribution function inequalities for martingales, Ann. Probability 1 (1973), 19-42. MR 0365692 (51 \#1944) 12

[CDV15] Ch. Cuny, J. Dedecker, and D. Volný, A functional CLT for fields of commuting transformations via martingale approximation, Zap. Nauchn. Sem. S.-Peterburg. Otdel. Mat. Inst. Steklov. (POMI) 441 (2015), no. Veroyatnost'i Statistika. 22, 239-262. MR 35045081

[Coh16] G. Cohen, A CLT for multi-dimensional martingale differences in a lexicographic order, Stochastic Process. Appl. 126 (2016), no. 5, 1503-1510. MR 34731031

[Cun15] Ch. Cuny, A compact LIL for martingales in 2-smooth Banach spaces with applications, Bernoulli 21 (2015), no. 1, 374-400. MR 3322323 2, 15, 18

[Ded01] J. Dedecker, Exponential inequalities and functional central limit theorems for a random fields, ESAIM Probab. Statist. 5 (2001), 77-104. MR 1875665 (2003a:60054) 1

[DGM10] J. Dedecker, S. Gouëzel, F. Merlevède, Some almost sure results for unbounded functions of intermittent maps and their associated Markov chains. Ann. Inst. Henri Poincaré Probab. Stat. 46 (2010), no. 3, 796-821. MR MR2682267 10

[EVW13] M. El Machkouri, D. Volný, and W. B. Wu, A central limit theorem for stationary random fields, Stochastic Process. Appl. 123 (2013), no. 1, 1-14. MR 2988107 2, 4

[FPS20] T. Fortune, M. Peligrad, and H. Sang, A Local Limit Theorem for Linear Random Fields, J. Time Ser. Anal.. https://doi.org/10.1111/jtsa.12556 3

[Gir19] D. Giraudo, Convergence rates in the central limit theorem for weighted sums of Bernoulli random fields, Mod. Stoch. Theory Appl. 6 (2019), no. 2, 251-267. MR 39638814

[Jia99] J. Jiang, Some laws of the iterated logarithm for two parameter martingales, J. Theoret. Probab. 12 (1999), no. 1, 49-74. MR 1674964 (2000e:60046) 2

[KVW16] J. Klicnarová, D. Volný, and Y. Wang, Limit theorems for weighted Bernoulli random fields under Hannan's condition, Stochastic Process. Appl. 126 (2016), no. 6, 1819-1838. MR 34837382

[LT91] M. Ledoux and M. Talagrand, Probability in Banach spaces, Ergebnisse der Mathematik und ihrer Grenzgebiete (3) [Results in Mathematics and Related Areas (3)], vol. 23, Springer-Verlag, Berlin, 1991, Isoperimetry and processes. MR 1102015 (93c:60001) 22

[MW11] A. Mallik and M. Woodroofe, A central limit theorem for linear random fields. Statist. Probab. Lett. 81 (2011), no. 11, 1623-1626. MR 28329213

[MP06] I. K. Matsak and A. M. Plīchko, A moment estimate for the supremum of normed sums in the law of the iterated logarithm, Ukraïn. Mat. Zh. 58 (2006), no. 5, 653-665. MR 22772882

[Nua09] D. Nualart, Malliavin calculus and its applications, CBMS Regional Conference Series in Mathematics, vol. 110, Published for the Conference Board of the Mathematical Sciences, Washington, DC; by the American Mathematical Society, Providence, RI, 2009. MR 249895322

[Pis76] G. Pisier, Sur la loi du logarithme itéré dans les espaces de Banach, Probability in Banach spaces (Proc. First Internat. Conf., Oberwolfach, 1975), 1976, pp. 203-210. Lecture Notes in Math., Vol. 526. MR 05012372

[PR198] S. Poghosyan and S. Rølly, Invariance principle for martingale-difference random fields, Statist. Probab. Lett. 38 (1998), no. 3, 235-245. MR 16299031

[PZ18a] M. Peligrad and N. Zhang, Martingale approximations for random fields, Electron. Commun. Probab. 23 (2018), Paper No. 28, 9. MR 37982391

[PZ18b] - On the normal approximation for random fields via martingale methods, Stochastic Process. Appl. 128 (2018), no. 4, 1333-1346. MR 37696641

[Sha06] A. P. Shashkin, The law of the iterated logarithm for an associated random field, Uspekhi Mat. Nauk 61 (2006), no. 2(368), 173-174. MR 22615542

[Sha15] Generalization of the law of the iterated logarithm for associated random fields, Mat. Zametki 98 (2015), no. 5, 769-781. MR 34385302 
[Vol15] D. Volný, A central limit theorem for fields of martingale differences, C. R. Math. Acad. Sci. Paris 353 (2015), no. 12, 1159-1163. MR 34279251

[VW14] D. Volný and Y. Wang, An invariance principle for stationary random fields under Hannan's condition, Stochastic Process. Appl. 124 (2014), no. 12, 4012-4029. MR 32644371

[Wic73] M. J. Wichura, Some Strassen-type laws of the iterated logarithm for multiparameter stochastic processes with independent increments, Ann. Probability 1 (1973), 272-296. MR 0394894 (52 \#15693) 1

[Wu05] W. B. Wu, Nonlinear system theory: another look at dependence, Proc. Natl. Acad. Sci. USA 102 (2005), no. 40, 14150-14154. MR 21722154 